\title{
AtTRB1-3 Mediates Structural Changes in AtPOT1b to Hold ssDNA
}

\author{
Amit Jaiswal \\ Centre for Bioinformatics, School of Life Sciences, Pondicherry University, Pondicherry 605014, India \\ Correspondence should be addressed to Amit Jaiswal; amitjai20@gmail.com
}

Received 31 October 2013; Accepted 19 November 2013; Published 16 February 2014

Academic Editors: V. Mathura and Y.-D. Park

Copyright (C) 2014 Amit Jaiswal. This is an open access article distributed under the Creative Commons Attribution License, which permits unrestricted use, distribution, and reproduction in any medium, provided the original work is properly cited.

POT from Arabidopsis thaliana is a member of shelterin complex and belongs to Telo_bind protein family. Three homologs are reported, namely, AtPOTla, AtPOT1b, and AtPOT1c, where AtPOT1b is involved in genomic stability and chromosome end protection by providing necessary grip to G-rich region of telomeric DNA for telomerase assembly. Telomeric binding factors (TRB1-3) physically interact with POT with no known functionality. In this work attempt has been made to elucidate the reason behind the interaction by analyzing molecular docking interaction between AtPOT1b and AtTRB1-3, which yielded potential residues, which could play essential role in structural modification. 3 ns molecular simulation helped to look into structural stability and conformational dynamics portraying domain movements. AtTRB's interaction with AtPOT1b provoked structural changes in AtPOT1b, thereby increasing the affinity for single strand DNA (ssDNA) as compared to double strand DNA (dsDNA). Although the obtained results require experimental evidence they can act as a guide in tracing the functions in other organisms. The information provided in this paper would be helpful in understanding functions of TRB1-3 with respect to genomic stability.

\section{Introduction}

Protection of telomere (POT1) is a single strand (ss) DNA binding protein which holds together the G-rich region of the telomeric sequence. To do such an intense job complexes of proteins have to join hands together. One such complex is shelterin which comprises of TRF1, TRF2, TIN2, TPP1, POT1, and RAP1 proteins. POT1 makes its presence in almost all eukaryotes being conserved by nature [1]. POT1 safeguards from rapid telomere loss and chromosome end fusion. Interruption of POT1 gene caused telomere recombination and severe DNA damage response [2]. Three POT1 homologs are identified in Arabidopsis, namely, POTla, POT1b, and POT1c, where POTla helps in telomere synthesis while POT1b is associated with chromosome end protection [3]. Baumann and Cech reported that POT1b directly binds with telomeric G-rich region in yeast [4]. However, POT1b of Arabidopsis does not follow aforementioned statement suggesting other proteins to be responsible for uniting the interaction with DNA [5]. Another group of SMH-like proteins (AtTRB13) binds specifically with telomeric DNA with unknown functionality [6]. Fascinatingly, physical association was also reported between AtPOT1b and AtTRB1-3 [7] which may be involved through Telo_bind domain $[8,9]$. Thus AtTRB1-3 might be a key player in arbitrating structural modifications in AtPOTlb to strongly hold DNA. Current research focuses on the interaction mediated by AtTRB1-3 to transform structural changes in AtPOT1b in order to bind ssDNA. Thus, in order to identify the molecular basis of interaction between AtPOT1b and AtTRB1-3 an attempt has been made using the molecular docking and molecular dynamics simulation technique.

\section{Materials and Methodology}

2.1. Sequence and Domain Analysis. Protein sequences of AtPOT1b, AtTRB1, AtTRB2, and AtTRB3 were collected from UniProt web server [10] (http://www.uniprot.org/) with their corresponding accession numbers Q6NKX5, Q8VWK4, Q9FJW5, and Q9M2X3, respectively. They were further subjected to InterProScan [11] (http://www.ebi.ac .uk/Tools/pfa/iprscan/) and ProtParam [12] (http://web.expasy.org/protparam/) for domain investigation and analyzing physico chemical properties. 
TABLE 1: Domain functioning screening for the proteins of choice.

\begin{tabular}{|c|c|c|c|c|c|c|c|}
\hline \multirow{3}{*}{$\begin{array}{l}\text { Server used } \\
\text { InterProScan }\end{array}$} & \multicolumn{7}{|c|}{ Proteins } \\
\hline & AtPOT1b & \multicolumn{2}{|c|}{ AtTRB1 } & \multicolumn{2}{|c|}{ AtTRB2 } & \multicolumn{2}{|c|}{ AtTRB3 } \\
\hline & $\begin{array}{l}\text { Telo_bind } \\
\text { domain } \\
(13-143)\end{array}$ & $\begin{array}{c}\text { SANT/Myb } \\
\text { domain }(5-55)\end{array}$ & $\begin{array}{l}\text { Histone H1/H5 } \\
\text { domain } \\
(123-182)\end{array}$ & $\begin{array}{c}\text { SANT/Myb } \\
\text { domain (5-55) }\end{array}$ & $\begin{array}{c}\text { Histone H1/H5 } \\
\text { domain } \\
(125-182)\end{array}$ & $\begin{array}{l}\text { SANT/Myb } \\
\text { domain } \\
(5-55)\end{array}$ & $\begin{array}{c}\text { Histone H1/H5 } \\
\text { domain } \\
(122-180)\end{array}$ \\
\hline
\end{tabular}

2.2. Tertiary Structure Prediction. Absence of three-dimensional structure in PDB database [13] (http://www.rcsb.org/) and lack of suitable templates for homology modeling made us employ I-TASSER [14] (http://zhanglab.ccmb.med.umich .edu/I-TASSER/) web application for protein structure prediction which is based on assembly simulations and multiple threading alignments by LOMETS [15]. The best model chosen was refined using What If server [16] (http:// swift.cmbi.ru.nl/servers/html/index.html) for removing bad contacts between protein atoms and was further subjected to molecular dynamics studies (MDS) using GROMACS 4.5.5 package for structural stability [17].

2.3. Molecular Dynamics Studies. GROMACS 4.5.5 package and Amber force field [18] were used to analyze model stability. The models were solvated with SPC-E water model using $0.9 \mathrm{~nm}$ triclinic box. The system was neutralized by replacing water molecules with counter ions sodium and chloride. The periodic boundary conditions were applied in all directions. Equilibration for both temperature $(300 \mathrm{~K})$ and pressure ( $1 \mathrm{~atm}$ ) was carried out for $100 \mathrm{ps}$ while the steepest descent algorithm was applied to 50,000 energy minimization steps for the constructed models. A total of 3000 ps (3 ns) production run was performed with a time step of $2 \mathrm{fs}$. The lowest potential energy conformations were selected from 3 ns MDS trajectory for further ProteinDNA interaction study. The model generated after MDS was subjected for validation using various web tools including PROCHECK [19], ERRAT [20], and VERIFY_3D [21], respectively.

2.4. Docking Studies. Single strand (ss) as well as double strand (ds) DNA with telomere sequence TTTAGGG starting from $5^{\prime}$ to $3^{\prime}$ was prepared using Discovery Studio software 3.1 (Discovery Studio Modeling Environment, Accelrys Software Inc., 2012). Three-dimensional structures derived from ITASSER web application were further used as input for HADDOCK web application specifying the Telo_bind domain (13143) of AtPOT1b and Histone H1/H5 domain (122-182) of AtTRB1-3 to dock in order to unearth the structural changes in AtPOT1b made by AtTRB1-3.

2.5. Docking of AtPOT1b with ssDNA. The least energy trajectory throughout $3 \mathrm{~ns}$ MDS of AtPOT1b was selected and docked with ssDNA using HADDOCK web application. Telo_bind domain of AtPOT1b was specified in input parameters and HADOCK score was considered for selecting best docked structure. The interacting residues were identified through Ligplot software [22].
2.6. Docking of AtPOT1b with AtTRB1-3. AtPOT1b was docked with AtTRB1-3 in order to find the structural changes in AtPOT1b induced by AtTRB1-3. The whole length domains of the protein were allowed to dock using HADDOCK web server forming three complexes, namely, AtPOT1b-AtTRB1, AtPOT1b-AtTRB2, and AtPOT1b-AtTRB3, respectively.

2.7. Docking AtPOT1b with ssDNA after Structural Changes. Structurally induced AtPOT1b was redocked with ssDNA through HADDOCK web application in order to find out whether H1/H5 domain of AtTRB1-3 induces Telo_bind domain to bind ssDNA. PRODY [23] application was used to track the domain movements.

\subsection{Docking AtPOT1b with dsDNA after Structural Changes.} In order to know the effect of AtTRB1-3 on AtPOT1b to bind dsDNA, structurally induced AtPOT1b was also redocked with dsDNA using HADDOCK web application. This also cross-validates the role of AtRB1-3 responsible for inducing AtPOT1b to bind either of ssDNA, dsDNA, or both.

\section{Results and Discussion}

3.1. Sequence and Domain Analysis. InterProScan predicted Telo_bind and $\mathrm{H} 1 / \mathrm{H} 5$ protein family to be present in AtPOT1b and AtTRB1-3. The residues belonging to the different families are described in Table 1. Telo_bind domain helps in recognition of single stranded DNA [8] while SANT/Myb domain also assists for DNA binding [24]. On the other hand, Histone $\mathrm{H} 1 / \mathrm{H} 5$ is responsible for protein interaction [25]. It gives a clear understanding that $\mathrm{H} 1 / \mathrm{H} 5$ domain should interact with Telo_bind domain in order to bring structural changes to bind to ssDNA more effectively.

ProtParam tool was used to analyze the molecular weight, isoelectric point, and amino acid percentage rich region of all the proteins. The properties analyzed revealed leucine (9\%) to be the most abundant amino acid in AtPOT1b followed by serine (7.9\%) and arginine (6.82\%). However, in AtTRB1-3 alanine was plenteous (12\%, 13.4\%, and $12.5 \%)$ in all, followed by glutamic acid (9\%) and serine $(8.7 \%)$ in AtTRB1 while lysine $(10.7 \%$ and $10.5 \%)$ and glutamic acid ( $8 \%$ and $8.5 \%)$ remained as the second and third highest in their frequency in both AtTRB2 and AtTRB3. Table 2 depicts physico chemical properties of all the proteins under investigation.

3.2. Tertiary Structure Prediction and Structural Analysis. ITASSER web application was used for deciphering the threedimensional structures as no suitable template was found 
TABLE 2: Physicochemical properties of concerned protein analysed through ProtParam.

\begin{tabular}{|c|c|c|c|c|}
\hline $\begin{array}{l}\text { Property } \\
\text { UniProt ID } \\
\end{array}$ & $\begin{array}{c}\text { AtPOT1b } \\
\text { (Q6NKX5) }\end{array}$ & $\begin{array}{c}\text { AtTRB1 } \\
\text { (Q8VWK4) }\end{array}$ & $\begin{array}{c}\text { AtTRB2 } \\
\text { (Q9FJW5) }\end{array}$ & $\begin{array}{c}\text { AtTRB3 } \\
\text { (Q9M2X3) } \\
\end{array}$ \\
\hline Number of amino acids & 454 & 300 & 299 & 295 \\
\hline Total number of atoms & 7376 & 4651 & 4685 & 4570 \\
\hline Molecular weight $(\mathrm{kDa})$ & 52.57 & 33.02 & 33.01 & 32.24 \\
\hline Theoretical pI & 8.38 & 9.34 & 9.87 & 9.52 \\
\hline Maximum $\%$ of residue & $\begin{array}{l}\text { Leucine (9) } \\
\text { Serine (7.9) } \\
\text { Arg }(6.8)\end{array}$ & $\begin{array}{l}\text { Alanine (12) } \\
\quad \text { Glu (9) } \\
\text { Serine (8.7) }\end{array}$ & $\begin{array}{l}\text { Alanine (13.4) } \\
\text { Lysine (10.7) } \\
\text { Glu (8) }\end{array}$ & $\begin{array}{l}\text { Alanine (12.5) } \\
\text { Lysine (10.5) } \\
\text { Glu (8.5) }\end{array}$ \\
\hline Minimum $\%$ of residue & Tryptophan (2) & Cysteine (1) & Cysteine (0.3) & Cysteine (0.3) \\
\hline $\begin{array}{l}\text { Total number of negatively charged } \\
\text { residues } \\
(\text { Asp + Glu) }\end{array}$ & 55 & 38 & 35 & 36 \\
\hline $\begin{array}{l}\text { Total number of positively charged } \\
\text { residues } \\
\text { (Arg + Lys) }\end{array}$ & 60 & 47 & 52 & 47 \\
\hline Instability index & 48.95 & 49.21 & 32.12 & 43.04 \\
\hline Aliphatic index & 85.84 & 72 & 72.98 & 74.88 \\
\hline Ext. coefficient (with Cys) & 68380 & 29575 & 31970 & 30940 \\
\hline Ext. coefficient (without Cys) & 67380 & 29450 & 31970 & 30940 \\
\hline Grand average of hydropathicity (GRAVY) & -0.215 & -0.623 & -0.623 & -0.558 \\
\hline
\end{tabular}

TABLE 3: List of templates used by I-TASSER for three-dimensional structure prediction.

\begin{tabular}{ll}
\hline $\begin{array}{l}\text { Protein } \\
\text { name }\end{array}$ & Templates \\
\hline POT1b & 2i0qA, 1jb7A, 1xjvA, 3kjpA \\
TRB1 & $\begin{array}{l}\text { 2osxA, 2lsoA, 1hstA, 4fsxA, 2juhA, 1h89A, 1hstA, } \\
\text { 1h88C, 3hfwA }\end{array}$ \\
TRB2 & 4fxgB, 2lsoA, 1hstA, 4fsxA, 2juhA, 1x58A, 1h88C, 4fxgB \\
TRB3 & 4fxgB, 1hstA, 1zrtD, 2juhA, 1x58A, 1h88C, 4fxgB, 2lsoA \\
\hline
\end{tabular}

for homology modeling. The various templates considered by I-TASSER server for structure prediction are mentioned in Table 3. The best structure was chosen based on $C$-score, TM score, and cluster density. C-score (confidence score) says about the significance about the threading template alignment. $C$-score is typically in the range of $[-5,2]$; thus, in our model the score was $-0.31,-3.24,-2.44$, and -2.20 for AtPOT1b, AtTRB1, AtTRB2, and AtTRB3, respectively. While expected TM score is scale for measuring the structural similarity between two structures and the range should be $>0.5$ indicating the model to be in correct topology [26], the TM score for the best model was found to be $0.67 \pm 0.13,0.55 \pm$ $0.12,0.53 \pm 0.14$, and $0.65 \pm 0.15$ which were in accordance with the above statement. A higher number of decoys 2089, 703,2315 , and 2336 for the modeled protein signify that the structure occurs more often in the simulation trajectory and therefore signifies a better quality model (Table 4). The best model was further taken for simulation and validation studies.

Detailed analysis of PDBsum server [27] revealed various important parameters like sheets, hairpins, psi loop, beta
TABLE 4: Identification of the best model generated based on the scoring pattern.

\begin{tabular}{|c|c|c|c|c|}
\hline $\begin{array}{l}\text { Protein } \\
\text { name }\end{array}$ & $\begin{array}{l}\text { I-TASSER } \\
\text { models }\end{array}$ & $C$-score & $\begin{array}{c}\text { Number } \\
\text { of } \\
\text { decoys }\end{array}$ & Cluster density \\
\hline \multirow{5}{*}{ POT1b } & Model $1^{*}$ & -0.31 & 2089 & 0.1410 \\
\hline & Model 2 & -2.20 & 314 & 0.0212 \\
\hline & Model 3 & -1.00 & 1049 & 0.0708 \\
\hline & Model 4 & -3.27 & 108 & 0.0073 \\
\hline & Model 5 & -3.69 & 71 & 0.0048 \\
\hline \multirow{5}{*}{ TRB1 } & Model $1^{*}$ & -3.24 & 703 & 0.0242 \\
\hline & Model 2 & -3.37 & 621 & 0.0214 \\
\hline & Model 3 & -3.66 & 461 & 0.0159 \\
\hline & Model 4 & -3.98 & 335 & 0.0115 \\
\hline & Model 5 & -4.19 & 272 & 0.0094 \\
\hline \multirow{5}{*}{ TRB2 } & Model $1^{*}$ & -2.44 & 2315 & 0.0533 \\
\hline & Model 2 & -4.67 & 251 & 0.0058 \\
\hline & Model 3 & -4.73 & 235 & 0.0054 \\
\hline & Model 4 & -4.90 & 199 & 0.0046 \\
\hline & Model 5 & -5.00 & 175 & 0.0040 \\
\hline \multirow{5}{*}{ TRB3 } & Model 1* & -2.20 & 2336 & 0.0696 \\
\hline & Model 2 & -3.54 & 611 & 0.0182 \\
\hline & Model 3 & -4.69 & 193 & 0.0058 \\
\hline & Model 4 & -4.82 & 169 & 0.0050 \\
\hline & Model 5 & -4.86 & 162 & 0.0048 \\
\hline
\end{tabular}

${ }^{*}$ Represents the best models taken into consideration generated by ITASSER server. 
TABLE 5: Various secondary structures predicted by PDBsum server.

\begin{tabular}{lccccccccc}
\hline $\begin{array}{l}\text { Protein } \\
\text { name }\end{array}$ & $\begin{array}{c}\text { Number of } \\
\text { sheet }\end{array}$ & $\begin{array}{c}\text { Number of } \\
\text { beta hairpins }\end{array}$ & $\begin{array}{c}\text { Number of } \\
\text { psi loop }\end{array}$ & $\begin{array}{c}\text { Number of } \\
\text { beta bulges }\end{array}$ & $\begin{array}{c}\text { Number of } \\
\text { strand }\end{array}$ & $\begin{array}{c}\text { Number of } \\
\text { helices }\end{array}$ & $\begin{array}{c}\text { Number of } \\
\text { helix-helix } \\
\text { interaction }\end{array}$ & $\begin{array}{c}\text { Number of } \\
\text { beta } \\
\text { turns }\end{array}$ & $\begin{array}{c}\text { Number of } \\
\text { gamma } \\
\text { turns }\end{array}$ \\
\hline AtPOT1b & 7 & 7 & 1 & 5 & 19 & 7 & 1 & 60 & 10 \\
AtTRB1 & None & None & None & None & None & 15 & 27 & 33 & 11 \\
AtTRB2 & 1 & 1 & None & None & 2 & 13 & 18 & 28 \\
AtTRB3 & 1 & 1 & None & 1 & 2 & 13 & 12 & 31 \\
\hline
\end{tabular}

TABLE 6: Evaluation of molecular dynamics studies for the chosen proteins.

\begin{tabular}{|c|c|c|c|c|}
\hline Protein & POT1B & TRB1 & TRB2 & TRB3 \\
\hline \multicolumn{5}{|c|}{ RMSD profile (from 3 ns MD trajectory) } \\
\hline Equilibration period (ps) & 250 & 2000 & 1200 & 2200 \\
\hline RMSD (nm) & 0.41 & 0.35 & 0.45 & 0.48 \\
\hline \multicolumn{5}{|l|}{ Potential energy $(\mathrm{kJ} / \mathrm{mol})$} \\
\hline Minimum & $\begin{array}{c}-2233890 \\
(2696 \text { frame })\end{array}$ & $\begin{array}{c}-796525.75 \\
\text { (1522 frame) }\end{array}$ & $\begin{array}{c}-988556.44 \\
\text { (2500 frame) }\end{array}$ & $\begin{array}{c}-912086.94 \\
\text { (1844 frame) }\end{array}$ \\
\hline Maximum & $\begin{array}{l}-2219754.8 \\
\text { (222 frame) }\end{array}$ & $\begin{array}{c}-788678.81 \\
\text { (8 frame) }\end{array}$ & $\begin{array}{c}-980101.88 \\
\text { (8 frame) }\end{array}$ & $\begin{array}{c}-903493.06 \\
\text { (14 frame) }\end{array}$ \\
\hline \multicolumn{5}{|c|}{ Ramachandran plot assessment } \\
\hline Residues in favored region (\%) & 80.5 & 80.1 & 83.8 & 84 \\
\hline Residues in additionally allowed region (\%) & 17 & 14.7 & 14.7 & 14.4 \\
\hline Residues in generously allowed region (\%) & 1.9 & 3.8 & 0.8 & 0.4 \\
\hline Residues in disallowed region (\%) & 0.5 & 1.5 & 0.8 & 1.2 \\
\hline Verify_3D score & 82.64 & 80.17 & 77.33 & 82.84 \\
\hline ERRAT score & 78.93 & 89.66 & 89.55 & 77.47 \\
\hline
\end{tabular}

bulges, strands, helices, helix-helix interaction, beta turns, and gamma turns for configuring the tertiary structure of the protein. AtTRB2 and AtTRB3 exhibited almost a common pattern in terms of the presence of sheets, beta hairpins, strands, and helices while AtTRB1 was totally different when compared to the other two. The results are shown in Table 5 and Figure 1.

Molecular structure of AtPOT1b shows Telo_bind domain (13-143), which is conserved throughout RPA like superfamily [3]. On the other hand, AtTRB1-3 shows two domains, namely, SANT/Myb domain (5-55) and histone H1/H5 domain (122-182). It has been reported that SANT/Myb domain usually binds to DNA [28] while H1/H5 binds to proteins [25], thereby suggesting a mechanism where H1/H5 domain binds with Telo_bind domain, thereby making structural changes in AtPOT1b. AtTRB-AtPOT1b complex then attaches itself to the single stranded telomeric G-rich strand due to the SANT/Myb domain present in AtTRB13. As the attachment is done with ssDNA, AtTRB (telomere repeat binding factor) gets rid of AtPOT1b making significant modification in the structural conformation of AtPOT1b.

3.3. Molecular Dynamics Studies (MDS) of Modeled Proteins. MDS was carried out for 3000 pico second ( $3 \mathrm{~ns}$ ) in order to evaluate the stability of the best I-TASSER models. Molecular dynamics studies gave insight into potential energy,
RMSD, and RMSF of three-dimensional structures. Both two-dimensional and three-dimensional structure predicted by I-TASSER server were evaluated after undergoing $3 \mathrm{~ns}$ simulation as seen in Figure 1. The minimum and maximum potential energies of all the proteins are listed in Table 6.

Root mean square deviation (RMSD) profiles are described in Figures 2(a)-2(d), which showed that the RMSD trajectory of AtPOT1b rises during the first $250 \mathrm{ps}$ (equilibration period) and remains quite stable during the following period with an average RMSD of $0.41 \mathrm{~nm}$, while TRB1, TRB2, and TRB3 stabilized after equilibration period of 2000 ps, 1200 ps, and 2200 ps with average RMSD asset values of $0.35 \mathrm{~nm}, 0.45 \mathrm{~nm}$, and $0.48 \mathrm{~nm}$, respectively. The overall residue fluctuation of each protein during the $3 \mathrm{~ns}$ timescale of the molecular dynamics simulation was also calculated and is described in Figures 2(e)-2(h) for AtPOT1b, AtTRB1, AtTRB2, and AtTRB3, respectively. RMSF results indicated that AtPOT1b protein has 37-43 $(0.1-0.21 \mathrm{~nm}), 54-56(0.1-0.18 \mathrm{~nm}), 85-94(0.1-0.27 \mathrm{~nm}), 113-$ $117(0.15-0.24 \mathrm{~nm})$, and $122-126(0.1-0.21 \mathrm{~nm})$ residues for Telo_bind domain (47-140). However, in AtTRB1 positions between $147-153(0.13-0.19 \mathrm{~nm})$ and $177-180(0.12-0.17 \mathrm{~nm})$, respectively, showed high flexibility. For AtTRB2 the fluctuating residues were 116-124 (0.12-0.15 nm) and 158-160 $(0.1-0.13 \mathrm{~nm})$ while for AtTRB3 the fluctuating residues are 105- $114(0.1-0.2)$ and $115-125(0.1-0.35 \mathrm{~nm})$, respectively. The above-mentioned regions of protein play vital role in 


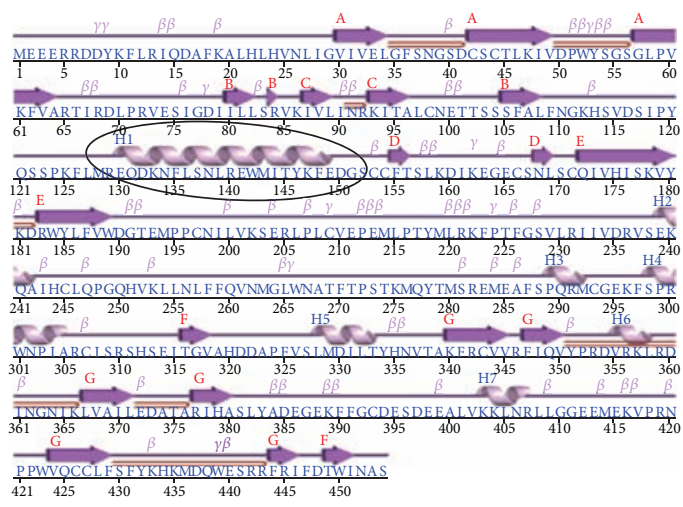

(a)

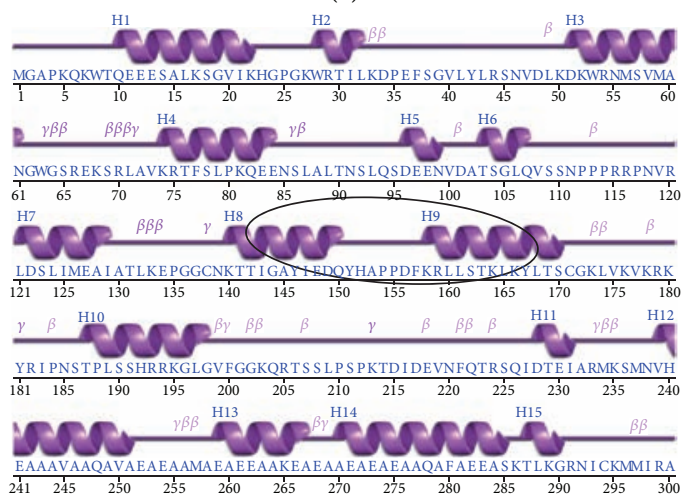

(b)
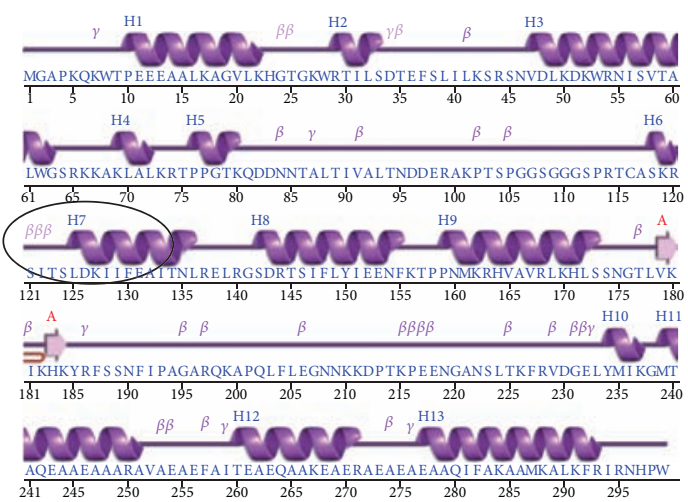

(c)
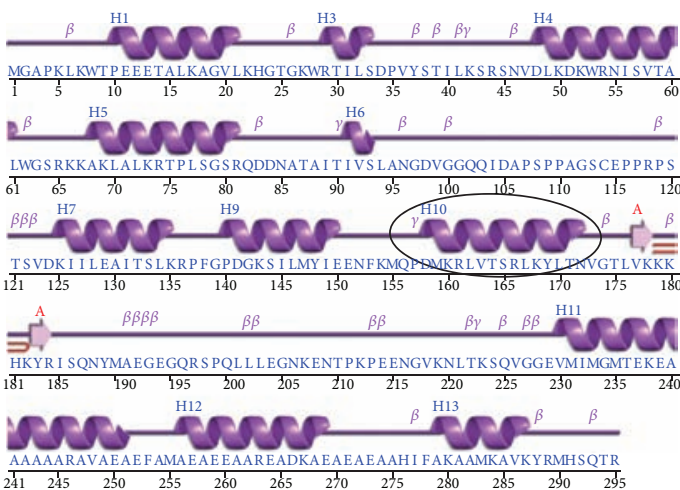

(d)

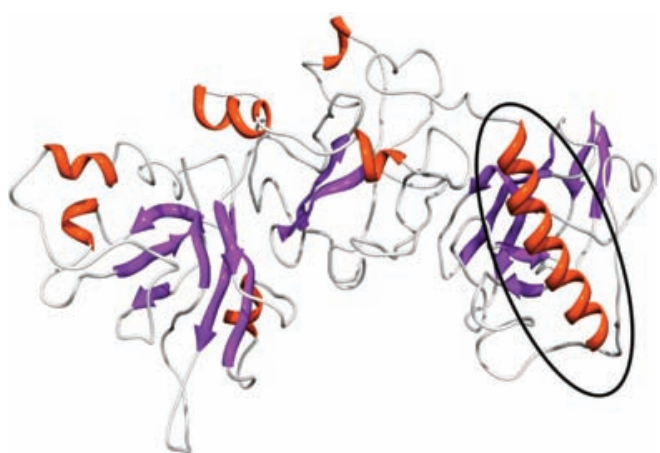

(e)

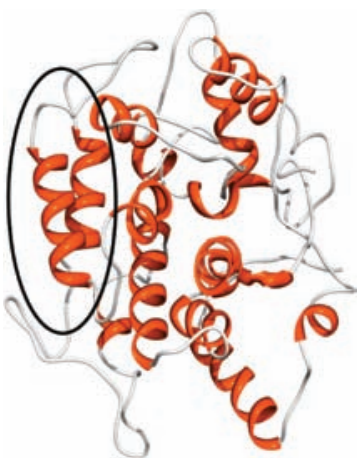

(f)

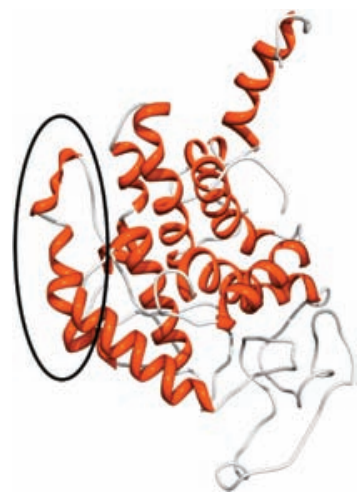

(g)

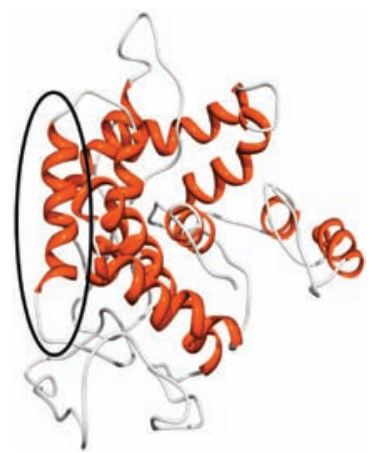

(h)

FIGURE 1: Two- and three-dimensional structures of best I-TASSER models after 3 ns simulation. 2D figures were generated in PDBsum server while 3D was prepared using Chimera software. ((a) and (e)) represent AtPOT1b; ((b) and (f)) represent AtTRB1; ((c) and (g)) represent AtTRB2; $((\mathrm{d})$ and $(\mathrm{h}))$ represent AtTRB3. Oval dashed lines represent the specific regions of protein which interact with each other for all the three proteins while the positions of interacting residues can be inferred from 2D figures. 

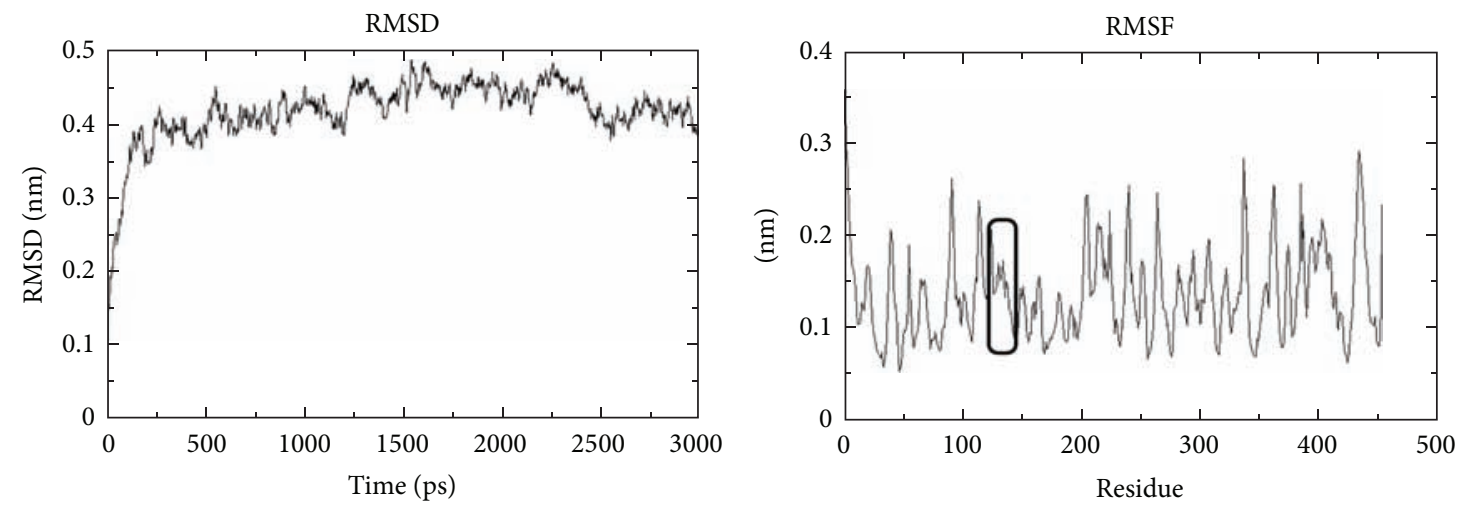

(a)
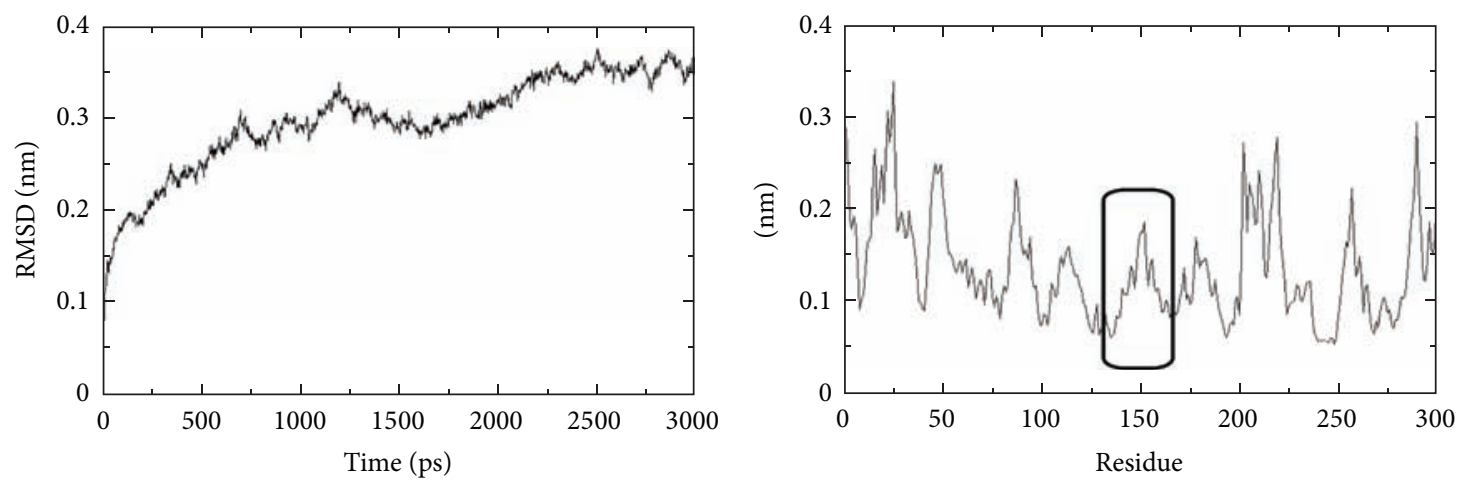

(b)
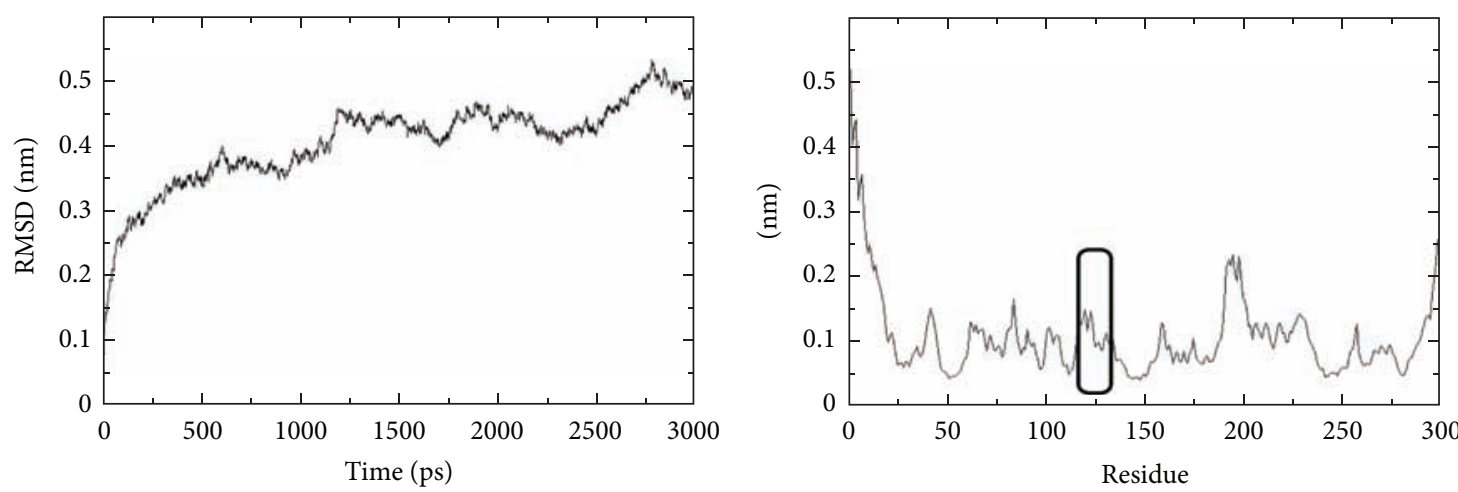

(c)

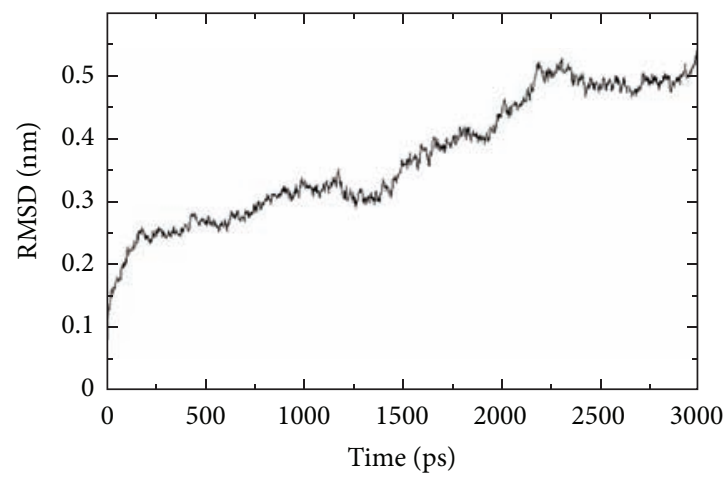

(d)

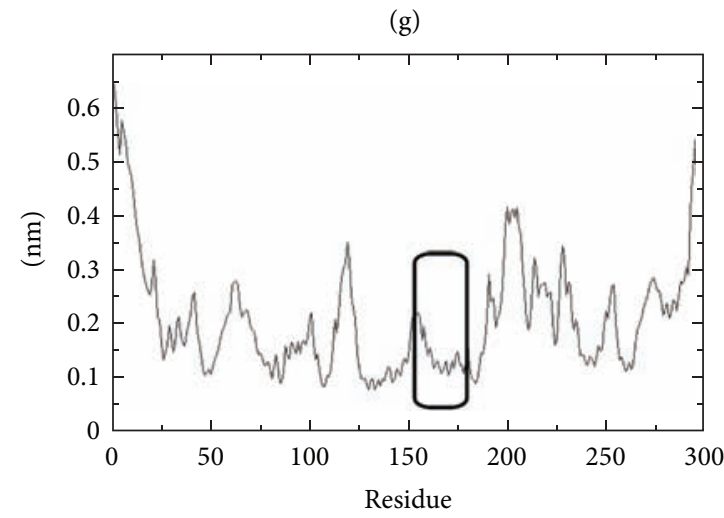

(h)

FiguRE 2: MDS trajectory based analysis of proteins under investigation. ((a)-(d)) represent RMSD profiles while ((e)-(h)) represent RMSF profiles of AtPOT1b, AtTRB1, AtTRB2, and AtTRB3, respectively. RMSDs of $\mathrm{C} \alpha$ atoms with respect to the initial structures of the proteins showed the stability of the model after the initial equilibration time. Oval dashed lines are the residues which were mostly involved in interaction, showing fewer fluctuations, suggesting the interactions to be stable. 


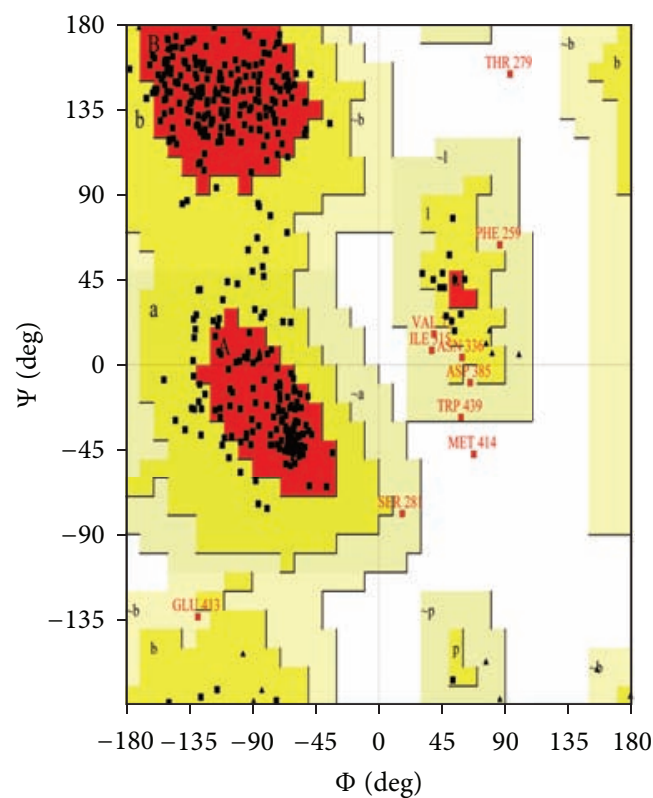

(a)

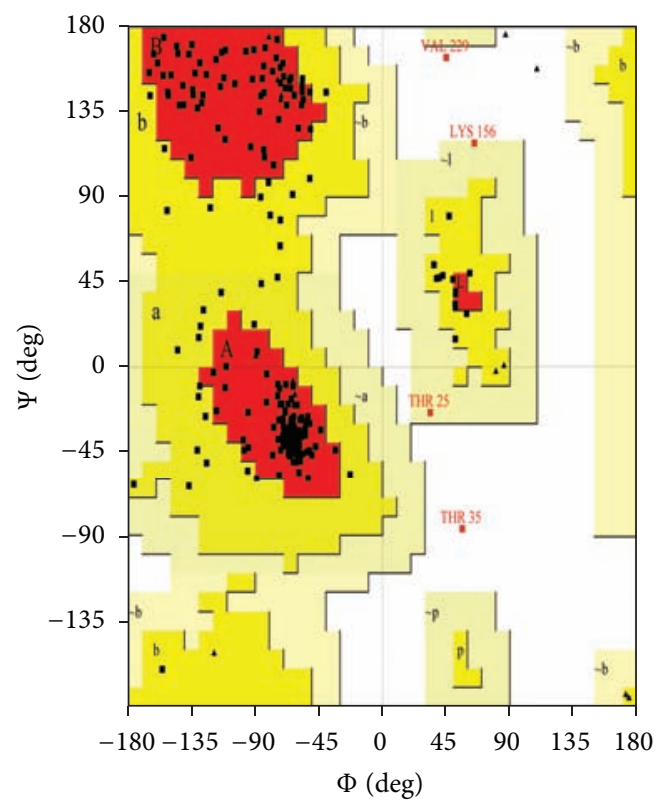

(c)

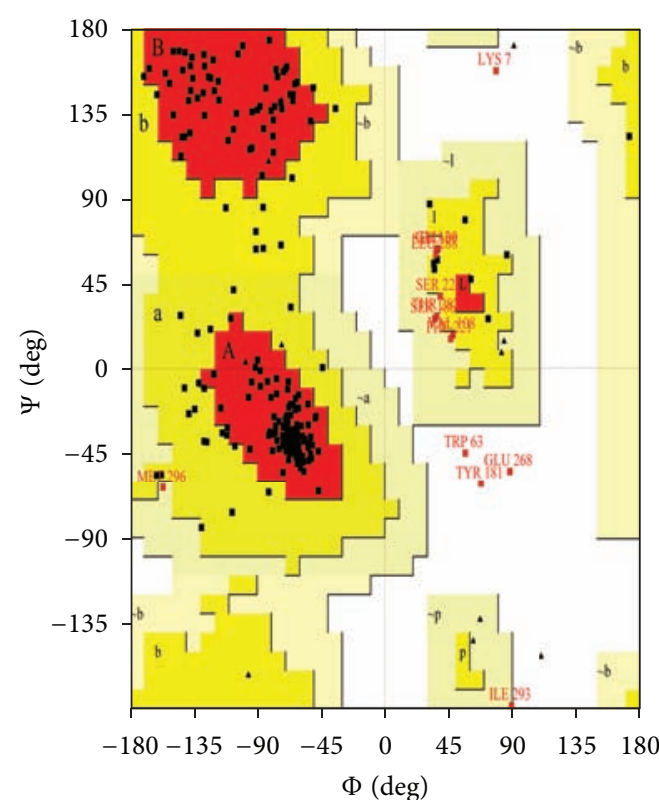

(b)

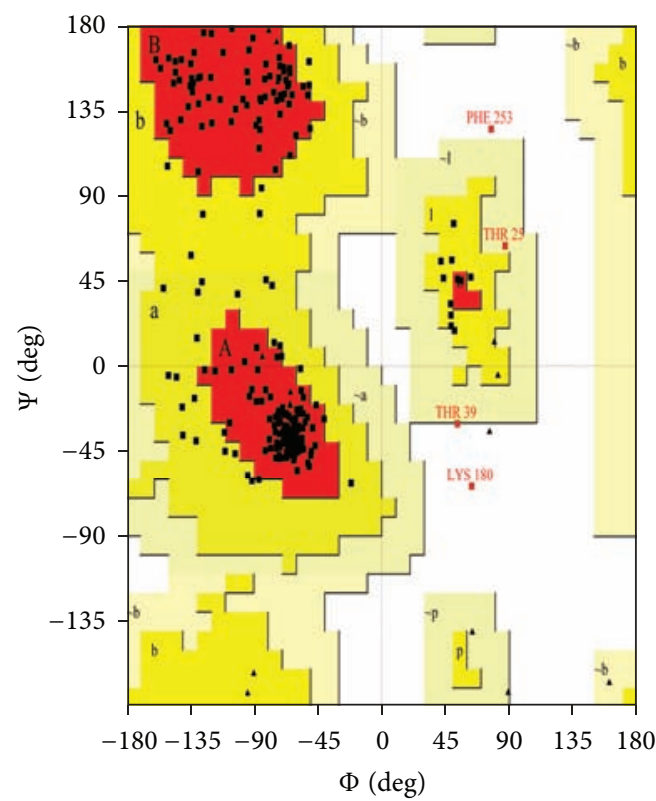

(d)

FIGURE 3: Ramachandran plot of the four proteins as depicted by PROCHECK server. Most favored regions are colored red, additionally allowed as yellow, generously allowed as light yellow, and disallowed regions as white fields, respectively.

the interactions by maintaining the conformational changes during the 3 ns molecular dynamics and can be observed in Figure 2.

3.4. Validation of the $3 D$ Structure. Molecular dynamics helped to increase the quality of protein structure. The stereochemical qualities of the 3 ns simulated models of the four proteins were validated by subjecting PDB files to structural analysis and verification server (SAVES) for Ramachandran plot, ERRAT score, and verify3D analysis, which showed that all model, had good quality factors and were reliable for further studies. Ramachandran plot represents red areas which correspond to the core regions representing the most favorable combination of phi-psi values [29]. Maximum likelihood for finding the protein residues $(>90 \%)$ in the core regions suggests better stereochemical quality. The results of PROCHECK analysis indicate that a relatively low percentage of residues have phi/psi angles in the disallowed regions suggesting the acceptability of Ramachandran plots for the four proteins. The percentage of allowed residues in the core region were found to be $97.5,94.8,98.5$, and $98.4 \%$ 


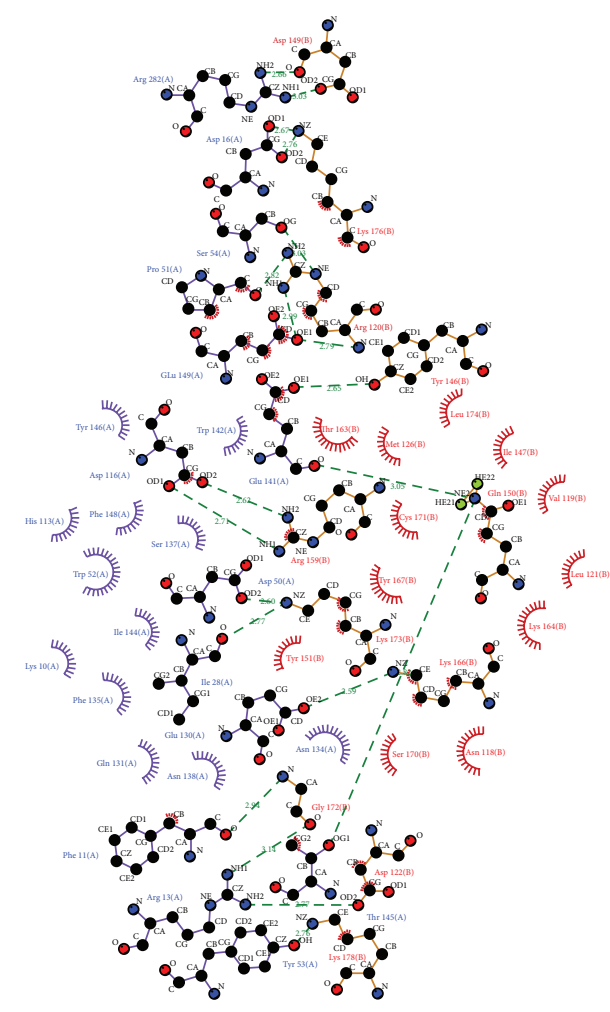

(a)

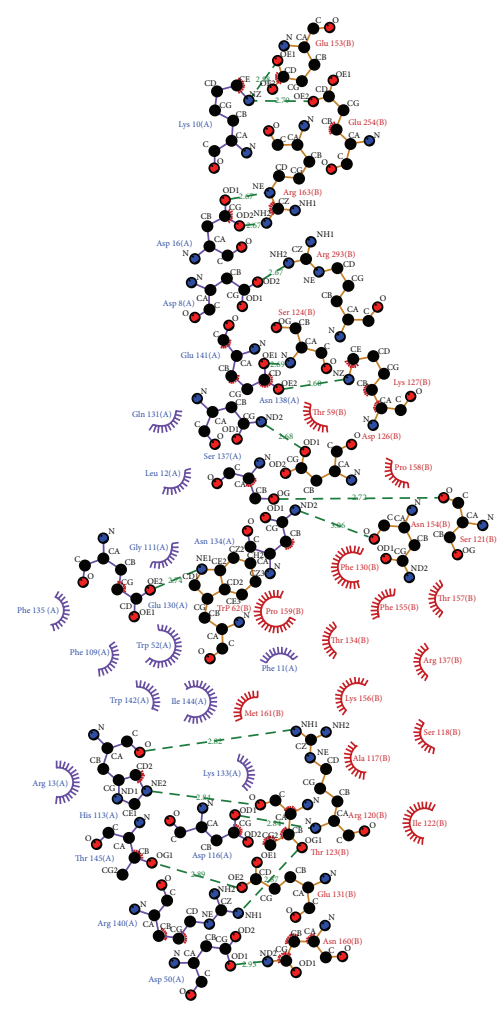

(b)

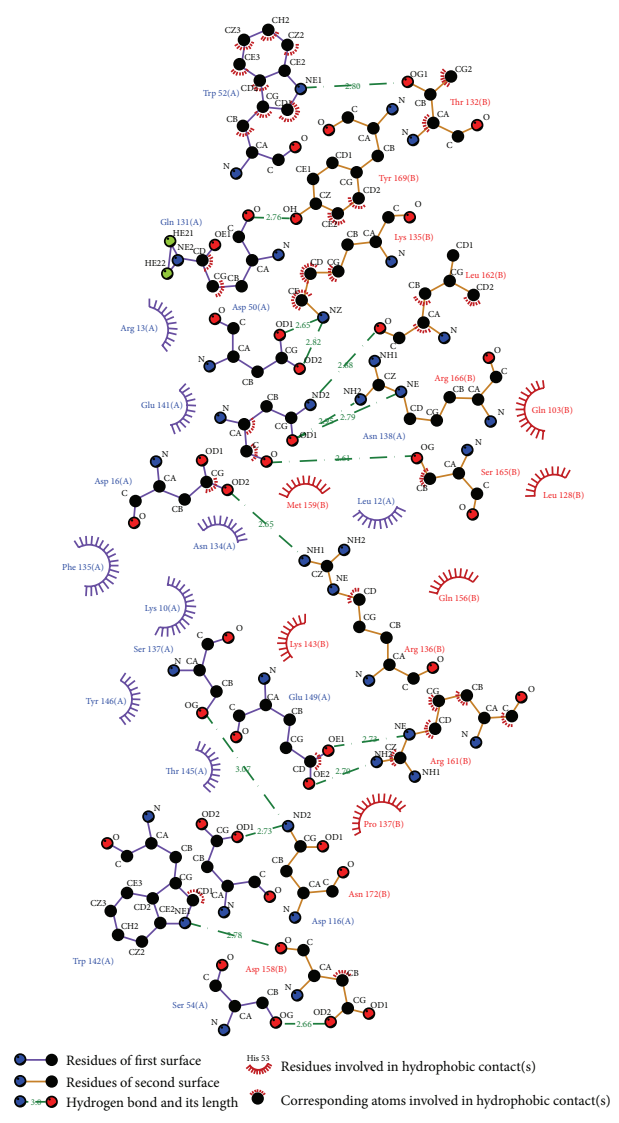

(c)

Figure 4: (a) DIMPLOT result of AtPOT1b-AtTRB1 interaction. Blue labels represent interacting residues of AtPOT1b while red ones represent AtTRB1. (b) DIMPLOT result of AtPOT1b-AtTRB2 interaction. Blue labels represent interacting residues of AtPOT1b while red ones represent AtTRB2. (c) DIMPLOT result of AtPOT1b-AtTRB3 interaction. Blue labels represent interacting residues of AtPOT1b while red ones represent AtTRB3.

and residues in the disallowed regions were found to be 0.5, 1.5, 0.8, and 1.2\% for AtPOT1b, AtTRB1, AtTRB2, and AtTRB3, respectively. The above-mentioned results can be seen from Table 6 and Figure 3. From the consequence, the stereochemical quality of the predicted model was found to be satisfactory.

3.5. AtPOT1b and ssDNA Interactions. The lowest potential energy of AtPOT1b was chosen to dock with ssDNA. Telo_bind domain of AtPOT1b was docked with ssDNA using HADDOCK web application. Haddock score for the complex was -1.7 while RMSD was $0.925 \AA$ which seemed to be quite significant in terms of structural changes made by ssDNA as depicted from Table 7 . The important residues which contributed in hydrogen bond formation with ssDNA are T66, R68, S103, F106, K125, L127, K222, H251, and K253 of which residues T66, R68, S103, F106, K125, and L127 belong to Telo_bind domain. Hydrogen bond plays a significant role which is very essential for three-dimensional structure [30]. Interacting residues are mentioned in Table 7.

3.6. Docking of AtPOT1b and AtTRB1-3. The lowest potential energy of each trajectory for four proteins was chosen and
TABLE 7: Interacting residues involved in AtPOT1b-ssDNA complex without the influence of AtTRB1-3.

\begin{tabular}{lc}
$\begin{array}{l}\text { Interacting residues of AtPOT1b } \\
\text { with ssDNA }\end{array}$ & $\begin{array}{c}\text { Hydrogen bond length } \\
(\AA)\end{array}$ \\
\hline A: THR66-B: GUA19 & $\mathbf{2 . 0 4 0 9 5}$ \\
${ }^{*}$ A: ARG68-B: GUA20 & $\mathbf{2 . 0 6 5 2 1}$ \\
${ }^{*}$ A: SER103-B: GUA19 & $\mathbf{2 . 0 5 7 9 3}$ \\
A: PHE106-B: GUA20 & $\mathbf{1 . 9 9 2 1 2}$ \\
${ }^{*}$ A: LYS125-B: THY22 & $\mathbf{1 . 9 7 9 5 8}$ \\
A: LEU127-B: GUA21 & $\mathbf{2 . 1 2 0 5 8}$ \\
A: LYS222-B: GUA12 & 2.21105 \\
A: LYS222-B: GUA12 & 2.13035 \\
A: LYS222-B: GUA13 & 2.08481 \\
A: HIS251-B: GUA12 & 2.4708 \\
A: LYS253-B: ADE11 & 2.12659 \\
\hline
\end{tabular}

Bold represents the residues involved in Telo_bind domain while * represents the common amino acids involved in AtPOT1b-ssDNA complex with and without AtTRB1-3 interaction.

the protein-protein interactions were performed using HADDOCK server. Since AtPOT1b significantly interacted with 
TABLE 8: List of interacting residues involved in three complexes, namely, AtPOT1b-AtTRB1, AtPOT1b-AtTRB2, and AtPOT1b-AtTRB3.

\begin{tabular}{|c|c|c|c|c|c|c|}
\hline Residues & Chain & Position number & Residues & Chain & Position number & $\begin{array}{l}\text { Distance of } \\
\text { H-bond }(\AA)\end{array}$ \\
\hline \multicolumn{7}{|c|}{ AtPOT1b-AtTRB1 } \\
\hline PHE & $\mathrm{A}$ & 11 & GLY & $\mathrm{B}$ & 172 & 2.94 \\
\hline ARG & A & 13 & GLY & $\mathrm{B}$ & 172 & 3.14 \\
\hline ARG & A & 13 & ASP & B & 122 & 2.77 \\
\hline ASP & A & 16 & LYS & $\mathrm{B}$ & 176 & 2.67 \\
\hline ASP & A & 16 & LYS & B & 176 & 2.76 \\
\hline ILE & A & 28 & LYS & B & 173 & 2.77 \\
\hline ASP & $\mathrm{A}$ & 50 & LYS & $\mathrm{B}$ & 173 & 2.6 \\
\hline PRO & A & 51 & ARG & $\mathrm{B}$ & 120 & 2.82 \\
\hline TYR & A & 53 & LYS & $\mathrm{B}$ & 178 & 2.76 \\
\hline SER & A & 54 & ARG & $\mathrm{B}$ & 120 & 3.03 \\
\hline ASP & A & 116 & ARG & $\mathrm{B}$ & 159 & 2.71 \\
\hline ASP & A & 116 & ARG & $\mathrm{B}$ & 159 & 2.62 \\
\hline GLU & A & 130 & LYS & $\mathrm{B}$ & 166 & 2.59 \\
\hline GLU & A & 141 & TYR & $\mathrm{B}$ & 146 & 2.65 \\
\hline GLU & A & 141 & GLN & B & 150 & 3.05 \\
\hline THR & A & 145 & GLN & $\mathrm{B}$ & 150 & 2.82 \\
\hline GLU & $\mathrm{A}$ & 149 & ARG & $\mathrm{B}$ & 120 & 2.79 \\
\hline GLU & A & 149 & ARG & $\mathrm{B}$ & 120 & 2.99 \\
\hline $\mathrm{ARG}$ & A & 282 & ASP & $\mathrm{B}$ & 149 & 3.03 \\
\hline $\mathrm{ARG}$ & A & 282 & ASP & B & 149 & 2.66 \\
\hline \multicolumn{7}{|c|}{ AtPOT1b-AtTRB2 } \\
\hline ASP & A & 8 & ARG & B & 293 & 2.67 \\
\hline LYS & A & 10 & GLU & $\mathrm{B}$ & 153 & 2.88 \\
\hline LYS & A & 10 & GLU & $\mathrm{B}$ & 254 & 2.79 \\
\hline ASP & A & 16 & ARG & B & 163 & 2.67 \\
\hline ASP & A & 16 & ARG & B & 163 & 2.67 \\
\hline ASP & A & 50 & ASN & $\mathrm{B}$ & 160 & 2.93 \\
\hline HIS & $\mathrm{A}$ & 113 & THR & B & 123 & 2.84 \\
\hline HIS & A & 113 & ARG & $\mathrm{B}$ & 120 & 2.82 \\
\hline ASP & $\mathrm{A}$ & 116 & ARG & B & 120 & 2.84 \\
\hline GLU & A & 130 & TRP & $\mathrm{B}$ & 62 & 2.74 \\
\hline ASN & A & 134 & ASN & $\mathrm{B}$ & 154 & 3.06 \\
\hline SER & A & 137 & SER & $\mathrm{B}$ & 121 & 2.72 \\
\hline ASN & A & 138 & ASP & $\mathrm{B}$ & 126 & 2.68 \\
\hline ARG & A & 140 & THR & B & 123 & 2.87 \\
\hline GLU & A & 141 & SER & $\mathrm{B}$ & 124 & 2.69 \\
\hline GLU & A & 141 & LYS & B & 127 & 2.6 \\
\hline THR & $\mathrm{A}$ & 145 & GLU & $\mathrm{B}$ & 131 & 2.89 \\
\hline \multicolumn{7}{|c|}{ AtPOT1b-AtTRB3 } \\
\hline ASP & A & 16 & ARG & B & 136 & 2.65 \\
\hline ASP & A & 50 & LYS & B & 135 & 2.65 \\
\hline ASP & A & 50 & LYS & B & 135 & 2.82 \\
\hline TRP & A & 52 & THR & B & 132 & 2.8 \\
\hline
\end{tabular}


TABLE 8: Continued.

\begin{tabular}{|c|c|c|c|c|c|c|}
\hline Residues & Chain & Position number & Residues & Chain & Position number & $\begin{array}{l}\text { Distance of } \\
\text { H-bond }(\AA)\end{array}$ \\
\hline SER & A & 54 & ASP & B & 158 & 2.66 \\
\hline ASP & A & 116 & ASN & B & 172 & 2.73 \\
\hline GLN & A & 131 & TYR & B & 169 & 2.76 \\
\hline SER & $\mathrm{A}$ & 137 & ASN & B & 172 & 3.07 \\
\hline ASN & A & 138 & ARG & B & 166 & 2.79 \\
\hline ASN & A & 138 & ARG & B & 166 & 2.95 \\
\hline ASN & A & 138 & LEU & B & 162 & 2.88 \\
\hline ASN & A & 138 & SER & B & 165 & 2.61 \\
\hline TRP & A & 142 & ASP & B & 158 & 2.78 \\
\hline GLU & A & 149 & ARG & B & 161 & 2.73 \\
\hline GLU & A & 149 & ARG & B & 161 & 2.7 \\
\hline
\end{tabular}

TABLE 9: Statistical analysis of HADDOCK results for each complex.

\begin{tabular}{lcccc}
\hline $\begin{array}{l}\text { Protein-nucleotide } \\
\text { complex (ssDNA) }\end{array}$ & AtPOTlb-ssDNA & $\begin{array}{c}\text { AtTRB1 induced } \\
\text { AtPOT1b-ssDNA }\end{array}$ & $\begin{array}{c}\text { AtTRB2 induced } \\
\text { AtPOT1b-ssDNA }\end{array}$ & $\begin{array}{c}\text { AtTRB3 induced } \\
\text { AtPOT1b-ssDNA }\end{array}$ \\
\hline Haddock score & $-1.7 \pm 4.8$ & $-53.0 \pm 14.0$ & $-54.3 \pm 9.3$ & $-63.8 \pm 7.6$ \\
RMSD $(\AA)$ & 0.925 & 1.815 & 1.455 & 2.304 \\
Van der Waals energy $(\mathrm{kJ} / \mathrm{mol})$ & $-80.7 \pm 7.1$ & $-77.6 \pm 3.1$ & $-76.2 \pm 4.3$ & $-77.3 \pm 8.5$ \\
Electrostatic energy $(\mathrm{kJ} / \mathrm{mol})$ & $-576.9 \pm 66.4$ & $-659.2 \pm 10.1$ & $-672.2 \pm 12.3$ & $-659.3 \pm 82.1$ \\
Buried surface area $\left(\AA^{2}\right)$ & $1956.0 \pm 185.7$ & $2019.4 \pm 107.8$ & $1955.8 \pm 99.3$ & $2003.5 \pm 84.4$ \\
Binding energy $(\mathrm{kcal} / \mathrm{mol})$ & -11580.27 & -18431.65 & -17323.66 & -17513.71 \\
\hline Protein-nucleotide & AtPOT1b-dsDNA & AtTRB1 induced & AtTRB2 induced & AtTRB3 induced \\
complex $(\mathrm{dsDNA})$ & $-4.3 \pm 6.8$ & $-31.5 \pm 5.3$ & $-36.1 \pm 12.5$ & AtPOT1b-dsDNA \\
\hline Haddock score & $0.9 \pm 0.1$ & $5.1 \pm 0.1$ & $0.9 \pm 0.6$ & $-31.5 \pm 7.4$ \\
RMSD $(\AA)$ & $-84.1 \pm 3.9$ & $-86.8 \pm 5.5$ & $-77.0 \pm 6.2$ & $0.9 \pm 0.5$ \\
Van der Waals energy $(\mathrm{kJ} / \mathrm{mol})$ & $-606.6 \pm 30.6$ & $-402.5 \pm 31.8$ & $-637.4 \pm 29.8$ & $-64.8 \pm 3.2$ \\
Electrostatic energy $(\mathrm{kJ} / \mathrm{mol})$ & $1987.9 \pm 89.2$ & $1819.7 \pm 19.2$ & $1970.8 \pm 131.4$ & $-637.9 \pm 55.5$ \\
Buried surface area $\left(\AA^{2}\right)$ & -1421.9 & -1680.84 & -1767.63 & $1857.3 \pm 130.0$ \\
Binding energy $(\mathrm{kcal} / \mathrm{mol})$ & & & -1702.43 \\
\hline
\end{tabular}

AtTRB1-3 through Telo_bind domain (13-143) and histone H1/H5 superfamily domain (115-183), respectively, they were reported to engage for telomerase assessment $[8,25]$. In fact, whole length protein family residues were used as active residues to define ambiguous interaction restrains (AIR) from which the best 200 structures were clustered for each AtPOT1b-AtTRB1, AtPOT1b-AtTRB2, and AtPOT1b-AtTRB3 interaction based on their binding energies. HADDOCK analyses of the best cluster showed intermolecular energies of $-219.072,-210.047$, and $-213.927 \mathrm{kcal} / \mathrm{mol}$, respectively, with very low ( $<2 \AA$ ) RMSD deviations suggesting that the structure of each complex was very stable.

From Figures 4(a)-4(c) and Table 8, hydrogen bond length of different interacting residues was identified. Twelve residues of AtPOT1b interacted with AtTRB1 making a total of seventeen hydrogen bonds between them. Similarly, for the second complex (AtPOT1b with AtTRB2) seventeen hydrogen bonds were formed by thirteen residues, while for the third complex (AtPOT1b with AtTRB3), ten residues were involved to give a total of fifteen hydrogen bonds. Residues lying within 130 to 142 of AtPOT1b were found to be significantly involved in the interaction with all the three proteins of AtTRB as evidenced by hydrogen bond frequencies, thus proving this region to be highly active. Ligplot application revealed none of the residues to be involved in Telo_bind domain to be able to interact with both histone $\mathrm{H} 1 / \mathrm{H} 5$ domain and ssDNA.

\subsection{Redocking of Induced AtPOT1b to ssDNA. HADDOCK} score revealed the best protein-protein complex between AtPOT1b-AtTRB1, AtPOT1b-AtTRB2, and AtPOT1b-AtTRB3 and was again redocked with ssDNA. In order to know whether AtTRB1-3 had induced AtPOT1b, root mean square deviation (RMSD) was keenly observed between the initial structure and the AtTRB1-3 induced structure. It was very surprising that RMSD between the initial and induced was very high at $1.815 \AA, 1.455 \AA$, and $2.304 \AA$, respectively. Deviating so much from the initial structure ironically suggests 


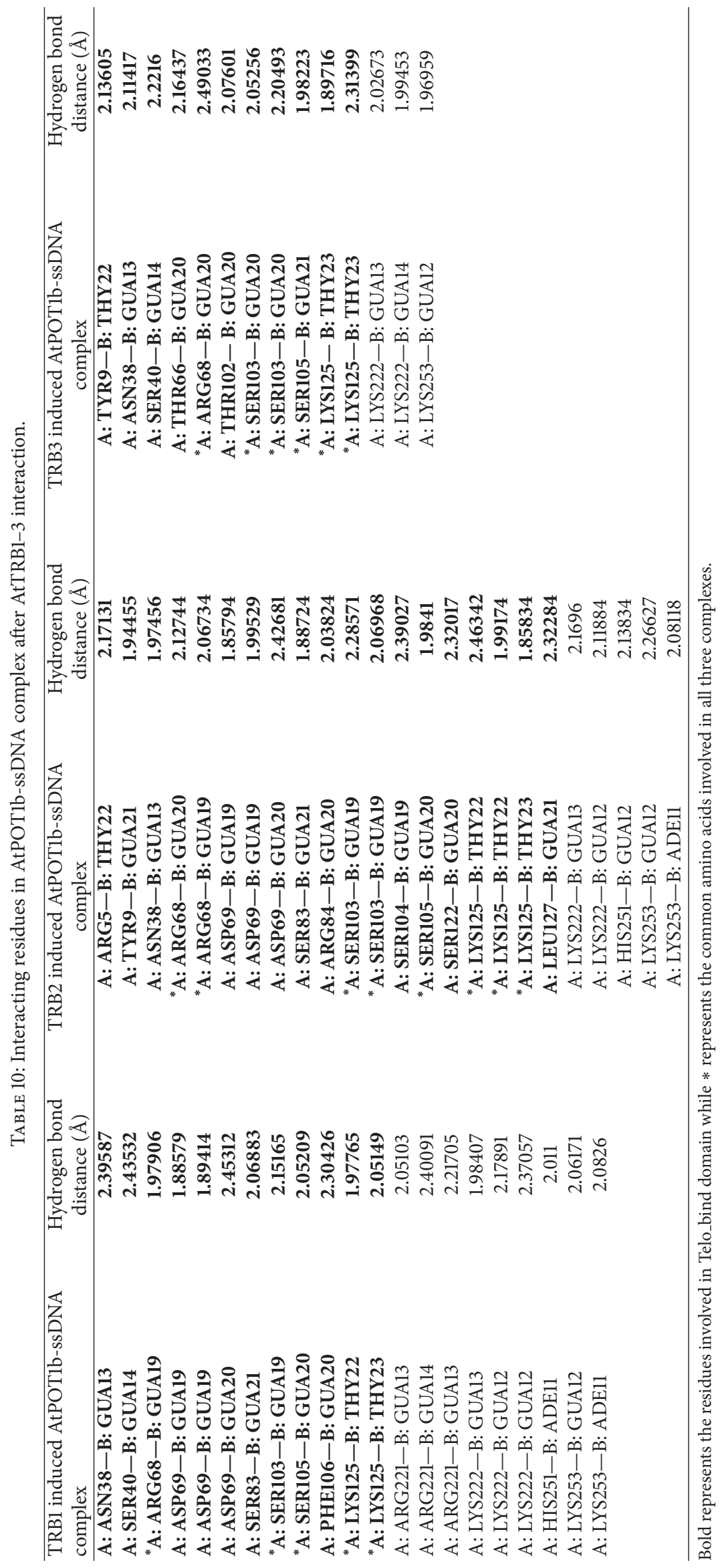




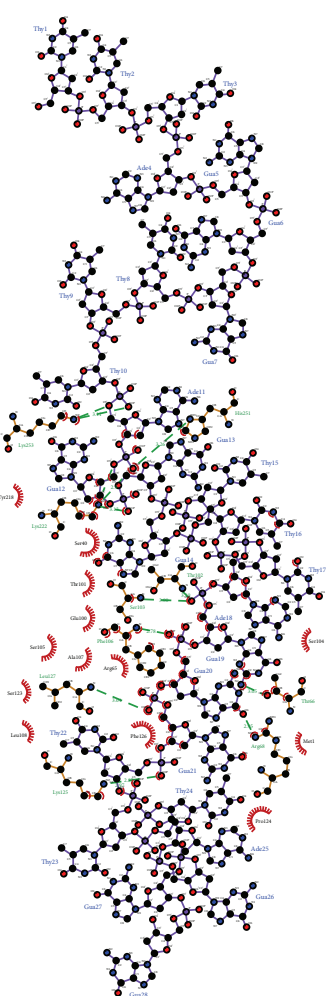

(a)

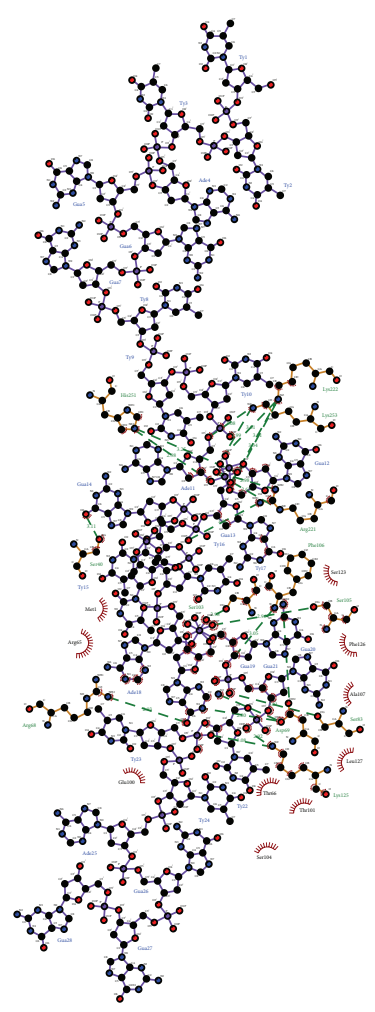

(b)

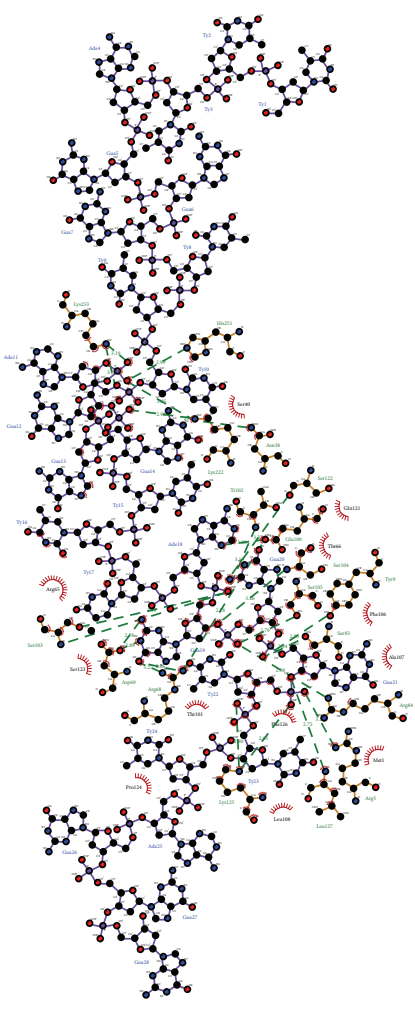

(c)

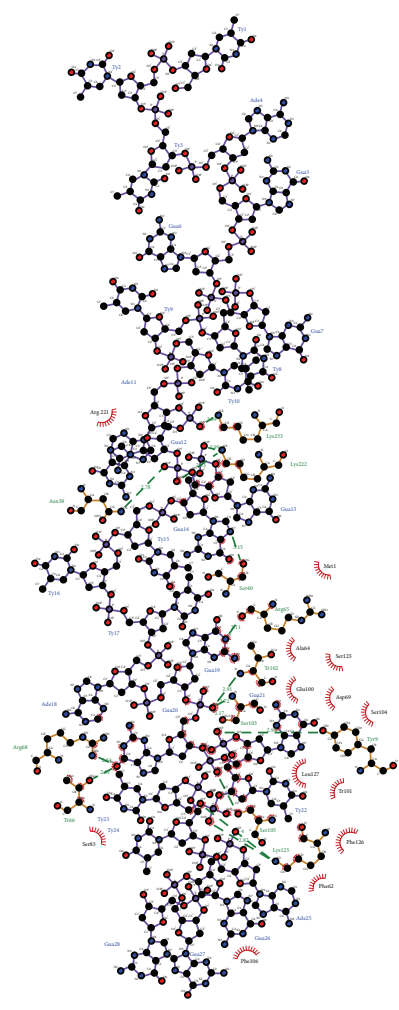

(d)

FIGURE 5: (a) LIGPLOT result of AtPOT1b-ssDNA without the influence of AtTRB1-3. (b) LIGPLOT result of AtPOT1b-ssDNA with the influence of AtTRB1. (c) LIGPLOT result of AtPOT1b-ssDNA with the influence of AtTRB2. (d) LIGPLOT result of AtPOT1b-ssDNA with the influence of AtTRB3 (green labels represent interacting residues of AtPOT1b while blue ones represent ssDNA).

that there is a significant change in the structure of AtPOT1b through the interaction of AtTRB1-3. HADDOCK score also increased from -1.7 to -63.8 as evidenced from Table 9 which also hints that AtTRB might be responsible for making structural changes in AtPOT1b in order to bind ssDNA more efficiently.

Interestingly, structural analysis revealed plenteous evidence to prove that AtTRB1-3 induces AtPOT1b. The total number of hydrogen bond increased to 21,24 , and 14 of which 12, 19, and 11 hydrogen bonds are due to the residues belonging to by Telo_bind domain owing to AtTRB1, AtTRB2, and AtTRB3 inducement. Two-dimensional and three-dimensional interactions between the proteins can be visualized from Figures 5(a)-5(d) and 6(a)-6(d), respectively, which are prepared using Ligplot [22] and Discovery Studio application 3.1 (Discovery Studio Modeling Environment, Accelrys Software Inc., 2012). The list of residues which were involved in hydrogen bond formation along with their bond length is mentioned in Table 10. It was clear from Tables 7 and 10 that R68, S103, and K125 are key amino acids present before and after AtTRB1-3 interaction. These key residues might play vital role in inducing structural changes as these are responsible for maintaining the interaction in all the proteinDNA complexes.

Moreover, stern look at the structure revealed the presence of three loops in triangular orientation governed by key amino acids, namely, R68, S103, and K125, respectively (Figure 8). Number of hydrogen bonds without AtTRB1-3 influence was three while with influence the bonds increased to six possibly stating the role of AtTRB1-3 in structural modification as depicted in Figures 7(a) and 7(b), respectively. As seen from the figure Ser 103 makes only one hydrogen bond while Ser 105 is responsible for none but as it is induced by AtTRB1-3 the number of hydrogen bonds increased for both residues (Table 10), illustrating the role of AtTRB1-3 in influencing structural changes. The presence of three amino acids, namely, Arg 68, Ser 103, and Lys 125, are responsible for the contraction of the three loops in triangular orientation in order to bind the ssDNA more firmly as seen in Figure 8. Residues R68, S103, and S105 bind guanine which supports the above-mentioned experimental evidence stating that POT1b specifically binds G-rich region of ssDNA. From the above evidences it is understood that AtTRB is responsible for inducing AtPOT1b where Ser 105 might be playing a decisive role in ssDNA attachment and vice versa.

PRODY interface [23] was used to compute anisotropic network model (ANM) calculations both before and after TRB1-3 interaction. ANM calculation provides the movements in the structures to be visualized. The movement direction of AtPOT1b due to AtTRB1-3 interaction can be visualized from Figures 9(a) and 9(b) which is represented with red arrows. Longer arrows indicate increased movement 


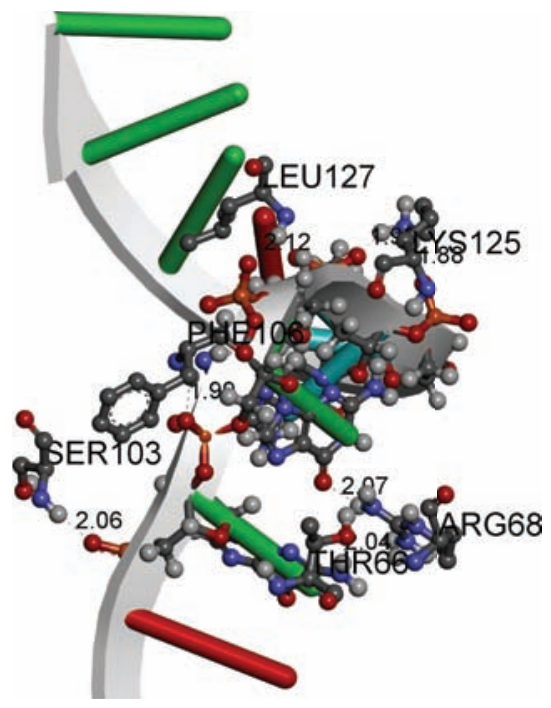

(a)

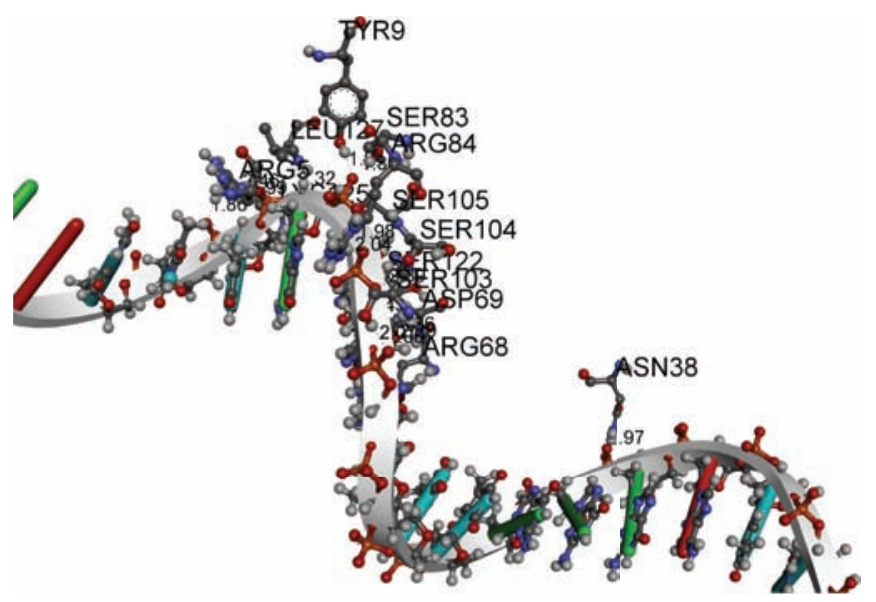

(c)

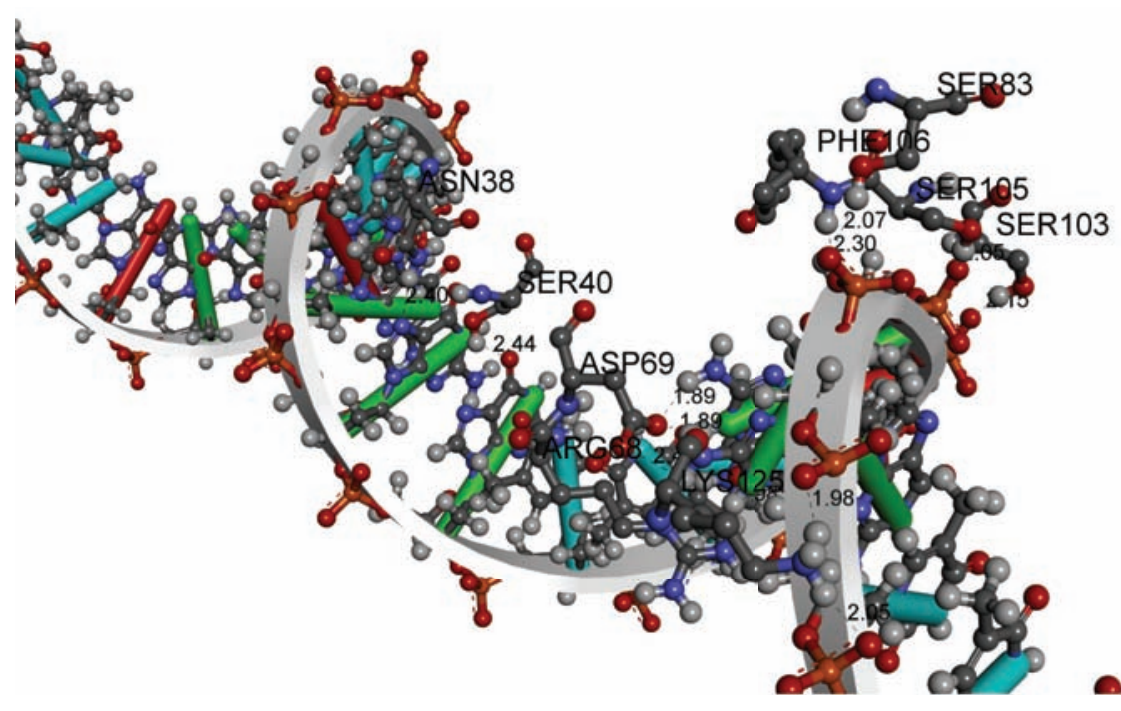

(b)

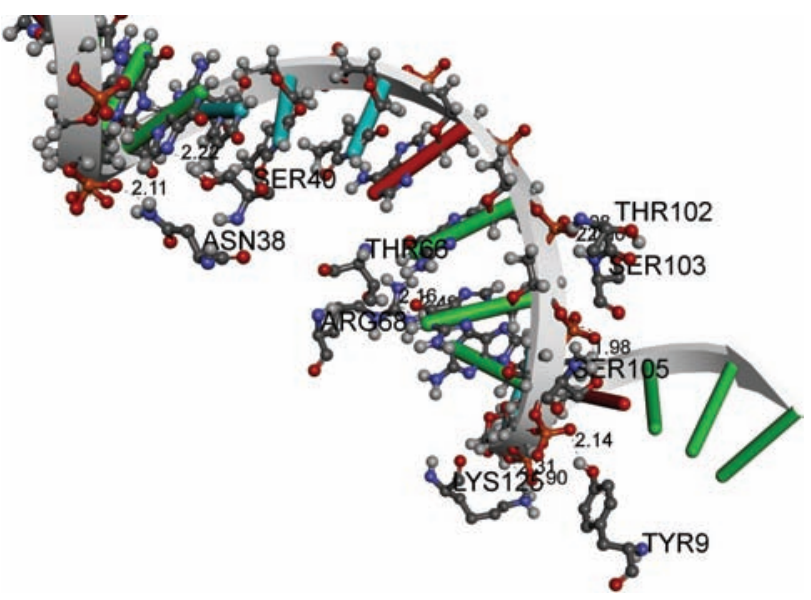

(d)

Figure 6: (a) Molecular docking of AtPOT1b-ssDNA without the influence of AtTRB1-3. (b) Molecular docking of AtPOT1b-ssDNA under the influence of AtTRB1. (c) Molecular docking of AtPOT1b-ssDNA under the influence of AtTRB2. (d) Molecular docking of AtPOT1bssDNA under the influence of AtTRB3. The interacting residues of AtPOT1b in each interaction belong to Telo_bind domain. Black dotted line represents hydrogen bond formation while the number denotes hydrogen bond length between them. The figure is represented in ball and stick model where blue, black, grey, and red balls represent nitrogen, carbon, hydrogen, and oxygen, respectively.

and displacement of AtPOT1b. Interestingly, the shorter arrows are pointing towards inward direction indicating the movement to be in closed conformation. However, in the case of TRB induced POTlb arrows are longer in length as well as pointing towards outer direction indicating an open conformation. The expansion of protein structure in an open form might be essential to firmly hold ssDNA. Binding energy score also supports the above statement (Table 9). AtTRB1-3 significantly increased the movement of Telo_bind domain in AtPOT1b leading to the conclusion that AtTRB13 definitely plays a vital role in bringing structural changes necessary for ssDNA attachment.

In order to confirm whether AtTRB1-3 induced AtPOT1b had any affinity towards dsDNA, HADDOCK web application was again used to cross-validate. AtPOT1b was docked with dsDNA. HADDOCK score and binding energy score were taken into consideration for interpreting the results which are depicted in Table 9. It was interesting to find insignificant HADDOCK scores of $-31.5,-36.1$, and -31.5 for AtTRB1-3 induced AtPOT1b when it was allowed to interact with dsDNA. The binding energy for ssDNA without the influence of AtTRB was $-11580.27 \mathrm{kcal} \mathrm{mol}^{-1}$ while under influence of AtTRB1, AtTRB2, and AtTRB3 the energy increased to $-18431.65,-17323.66$, and $-17513.71 \mathrm{kcal} \mathrm{mol}^{-1}$, respectively. With respect to dsDNA the binding energy with and without AtTRB1-3 influence was $-1680.84,-1767.63$, -1702.43 , and $-1421.9 \mathrm{kcal} \mathrm{mol}^{-1}$, respectively, as seen in Table 9 . The binding energy results were also in concordance with the said hypothesis.

Thus, it might be concluded that AtTRB1-3 interaction with AtPOTlb (Telo_bind domain) might induce the later to bind ssDNA more efficiently rather than dsDNA. Triangular 


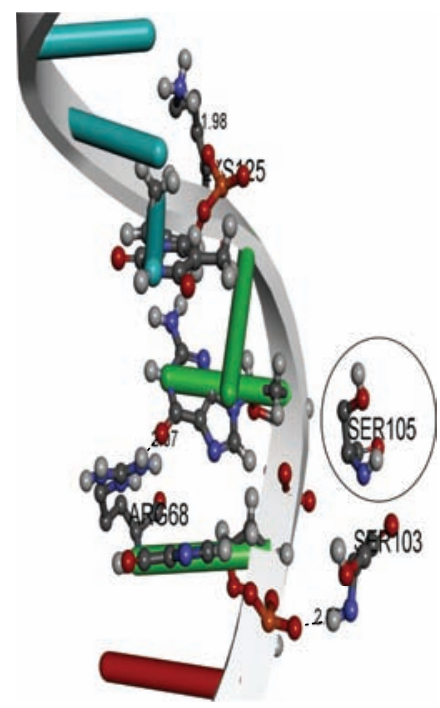

(a)

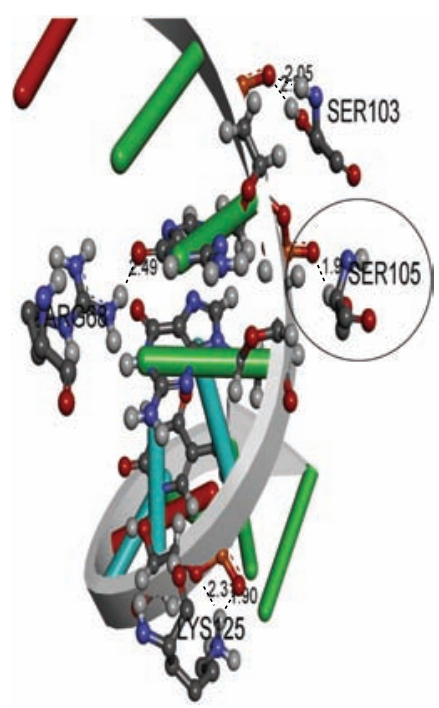

(b)

FIGURE 7: Molecular interaction of serine 105 with and without AtTRB's influence. Ser 105 is involved in forming one hydrogen bond with guanine under the influence of AtTRB as shown in (a) while none without influence as shown in (b). TRB is responsible to alter the orientation of Ser 105, thereby hindering hydrogen bond formation. Ser 105 is marked with a black circle while hydrogen bonds are represented as black dotted lines.

orientation of the three amino acids helps in providing stability to the telomeric single stranded DNA leading to efficient telomere elongation headed by telomerase.

\section{Conclusion}

AtPOT1b plays a very vital role in maintaining genomic stability with the help of transcription factors AtTRB1-3. Moreover, molecular model for AtPOT1b, AtTRB1, AtTRB2,

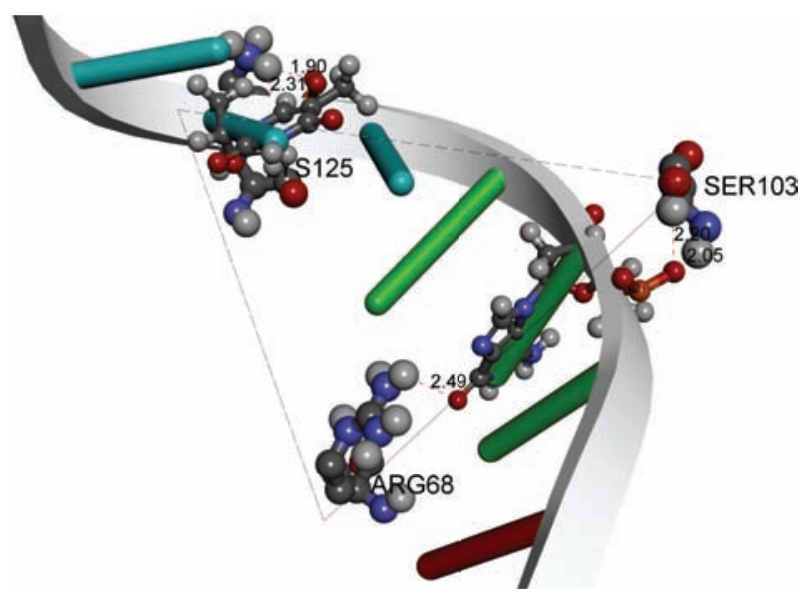

FIGURE 8: Triangular orientation of the key amino acids. R68, S103, and K125 can be seen in a triangular orientation which is responsible for hydrogen bond formation represented by red dotted lines along with their distances. The binding of these residues is responsible for conformational changes in AtPOT1b leading to a firm grip for ssDNA.

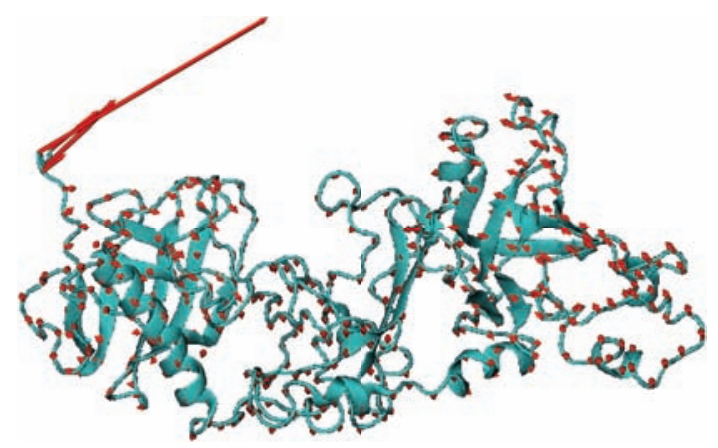

(a)

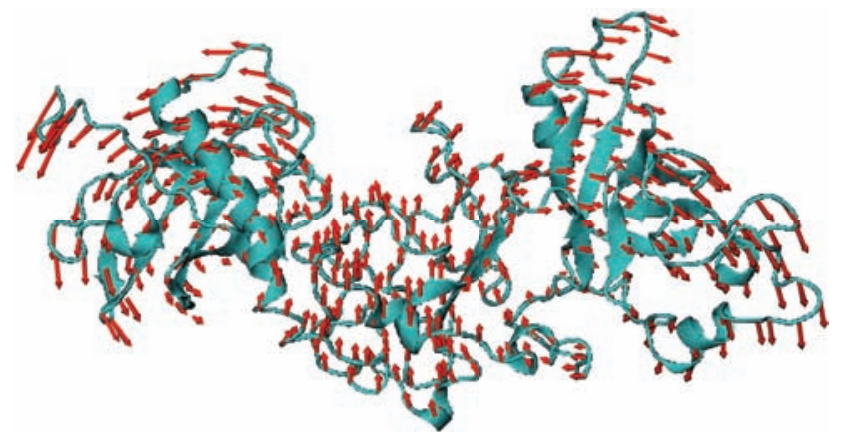

(b)

FIGURE 9: (a) Recessive motion observed in non-AtTRB1-3 influenced AtPOT1b. Structural transformations and movement can be depicted from the red arrows. The overall movement of AtPOTlb is very less as represented by shorter red arrows pointing inwards illustrating closed conformation of AtPOT1b. (b) Dominant motion observed in AtTRB1-3 influenced AtPOT1b. Structural transformations and movement can be depicted from the red arrows. The overall movement of AtPOT1b is very high as represented by longer red arrows pointing outwards illustrating open conformation of AtPOT1b. 
and AtTRB3 has been predicted and the role of AtTRB13 with respect to AtPOT1b was analyzed. The threedimensional structure was modeled using I-TASSER web application and their stability was analyzed through $3 \mathrm{~ns}$ molecular dynamics simulation studies. Structure validation was performed through Ramachandran plot, verify3D, and ERRAT scores. Molecular docking analysis helped to locate structural transitions brought in AtPOT1b through AtTRB13 interaction while structural analysis assisted in finding key residues, namely, R68, S103, and K125, to be responsible in mediating interaction with ssDNA. S105 on the other hand might be responsible for efficient gripping of ssDNA. RMSD scores of $1.815 \AA, 1.455 \AA$, and $2.304 \AA$ also supported the fact that AtTRB1-3 interacts with AtPOT1b mediating structural transformations in AtPOTlb in order to bind ssDNA more efficiently than dsDNA as portrayed from HADDOCK and binding energy scores. Moreover, the attachment to DNA is mediated by the key amino acids which form a triangular orientation in order to interact with ssDNA. Nevertheless, the order of AtTRB1-3 interaction is still unknown and future investigation is required in order to trace the transcriptional regulation of protection of telomeres (POT). More work will be needed to check whether the above-mentioned result is true for humans and other organisms.

\section{Conflict of Interests}

The author declares that there is no conflict of interests regarding the publication of this paper.

\section{Acknowledgment}

The author is grateful to Department of Biotechnology (DBT), Government of India, for providing training fellowship to carry out research at Centre for Bioinformatics, Pondicherry University, Pondicherry, India.

\section{References}

[1] T. de Lange, “Telomere capping: one strand fits all," Science, vol. 292, no. 5519, pp. 1075-1076, 2001.

[2] J. Z.-S. Ye, D. Hockemeyer, A. N. Krutchinsky et al., "POT1interaction protein PIP1: a telomere length regulator that recruits POT1 to the TIN2/TRF1 complex," Genes and Development, vol. 18, no. 14, pp. 1649-1654, 2004.

[3] E. V. Shakirov, Y. V. Surovtseva, N. Osbun, and D. E. Shippen, "The Arabidopsis Pot1 and Pot2 proteins function in telomere length homeostasis and chromosome end protection," Molecular and Cellular Biology, vol. 25, no. 17, pp. 7725-7733, 2005.

[4] P. Baumann and T. R. Cech, "Pot1, the putative telomere endbinding protein in fission yeast and humans," Science, vol. 292, no. 5519, pp. 1171-1175, 2001.

[5] E. V. Shakirov, T. D. McKnight, and D. E. Shippen, "POT1independent single-strand telomeric DNA binding activities in Brassicaceae," Plant Journal, vol. 58, no. 6, pp. 1004-1015, 2009.

[6] Z. N. Karamysheva, Y. V. Surovtseva, L. Vespa, E. V. Shakirov, and D. E. Shippen, "A C-terminal Myb extension domain defines a novel family of double-strand telomeric DNA-binding proteins in arabidopsis," Journal of Biological Chemistry, vol. 279, no. 46, pp. 47799-47807, 2004.

[7] P. P. Schrumpfová, M. Kuchař, J. Paleček, and J. Fajkus, "Mapping of interaction domains of putative telomere-binding proteins AtTRB1 and AtPOT1b from Arabidopsis thaliana," FEBS Letters, vol. 582, no. 10, pp. 1400-1406, 2008.

[8] R. M. Mitton-Fry, E. M. Anderson, T. R. Hughes, V. Lundblad, and D. S. Wuttke, "Conserved structure for single-stranded telomeric DNA recognition," Science, vol. 296, no. 5565, pp. 145$147,2002$.

[9] D. Churikov and C. M. Price, "Potl and cell cycle progression cooperate in telomere length regulation," Nature Structural and Molecular Biology, vol. 15, no. 1, pp. 79-84, 2008.

[10] A. Bairoch, R. Apweiler, C. H. Wu et al., "The Universal Protein Resource (UniProt)," Nucleic Acids Research, vol. 33, pp. D154D159, 2005.

[11] E. Quevillon, V. Silventoinen, S. Pillai et al., "InterProScan: protein domains identifier," Nucleic Acids Research, vol. 33, no. 2, pp. W116-W120, 2005.

[12] E. Gasteiger, C. Hoogland, A. Gattiker et al., "Protein identification and analysis tools on the ExPASy server," in The Proteomics Protocols Handbook, pp. 571-607, Springer, 2005.

[13] J. L. Sussman, D. Lin, J. Jiang et al., "Protein Data Bank (PDB): database of three-dimensional structural information of biological macromolecules," Acta Crystallographica D, vol. 54, no. 6, pp. 1078-1084, 1998.

[14] A. Roy, A. Kucukural, and Y. Zhang, "I-TASSER: a unified platform for automated protein structure and function prediction," Nature protocols, vol. 5, no. 4, pp. 725-738, 2010.

[15] A. Roy, A. Kucukural, and Y. Zhang, "I-TASSER: a unified platform for automated protein structure and function prediction," Nature protocols, vol. 5, no. 4, pp. 725-738, 2010.

[16] R. Rodriguez, G. Chinea, N. Lopez, T. Pons, and G. Vriend, "Homology modeling, model and software evaluation: three related resources," Bioinformatics, vol. 14, no. 6, pp. 523-528, 1998.

[17] S. Pronk, S. Páll, R. Schulz et al., "GROMACS 4. 5: a highthroughput and highly parallel open source molecular simulation toolkit," Bioinformatics, vol. 29, pp. 845-854, 2013.

[18] K. Lindorff-Larsen, S. Piana, K. Palmo et al., "Improved sidechain torsion potentials for the Amber ff99SB protein force field," Proteins, vol. 78, no. 8, pp. 1950-1958, 2010.

[19] R. A. Laskowski, M. W. MacArthur, D. S. Moss, and J. M. Thornton, "PROCHECK: a program to check the stereochemical quality of protein structures," Journal of Applied Crystallography, vol. 26, pp. 283-291, 1993.

[20] C. Colovos and T. O. Yeates, "Verification of protein structures: patterns of nonbonded atomic interactions," Protein Science, vol. 2, no. 9, pp. 1511-1519, 1993.

[21] J. U. Bowie, R. Luthy, and D. Eisenberg, "A method to identify protein sequences that fold into a known three-dimensional structure," Science, vol. 253, no. 5016, pp. 164-170, 1991.

[22] R. A. Laskowski and M. B. Swindells, "LigPlot+: multiple ligandprotein interaction diagrams for drug discovery," Journal of Chemical Information and Modeling, vol. 51, no. 10, pp. 27782786, 2011.

[23] A. Bakan, L. M. Meireles, and I. Bahar, "ProDy: protein dynamics inferred from theory and experiments," Bioinformatics, vol. 27, no. 11, Article ID btr168, pp. 1575-1577, 2011.

[24] R. Aasland, A. F. Stewart, and T. Gibson, "The SANT domain: a putative DNA-binding domain in the SWI-SNF and ADA 
complexes, the transcriptional co-repressor N-CoR and TFIIIB," Trends in Biochemical Sciences, vol. 21, no. 3, pp. 87-88, 1996.

[25] V. Ramakrishnan, J. T. Finch, V. Graziano, P. L. Lee, and R. M. Sweet, "Crystal structure of globular domain of histone H5 and its implications for nucleosome binding," Nature, vol. 362, no. 6417, pp. 219-223, 1993.

[26] Y. Zhang and J. Skolnick, "Scoring function for automated assessment of protein structure template quality," Proteins, vol. 57, no. 4, pp. 702-710, 2004.

[27] R. A. Laskowski, V. V. Chistyakov, and J. M. Thornton, "PDBsum more: new summaries and analyses of the known 3D structures of proteins and nucleic acids," Nucleic Acids Research, vol. 33, pp. D266-D268, 2005.

[28] R. Aasland, A. F. Stewart, and T. Gibson, "The SANT domain: a putative DNA-binding domain in the SWI-SNF and ADA complexes, the transcriptional co-repressor N-CoR and TFIIIB," Trends in Biochemical Sciences, vol. 21, no. 3, pp. 87-88, 1996.

[29] G. N. Ramachandran, C. Ramakrishnan, and V. Sasisekharan, "Stereochemistry of polypeptide chain configurations," Journal of Molecular Biology, vol. 7, pp. 95-99, 1963.

[30] G. G. R. Desiraju and T. Steiner, The Weak Hydrogen Bond: In Structural Chemistry and Biology, vol. 9, Oxford University Press, 2001. 

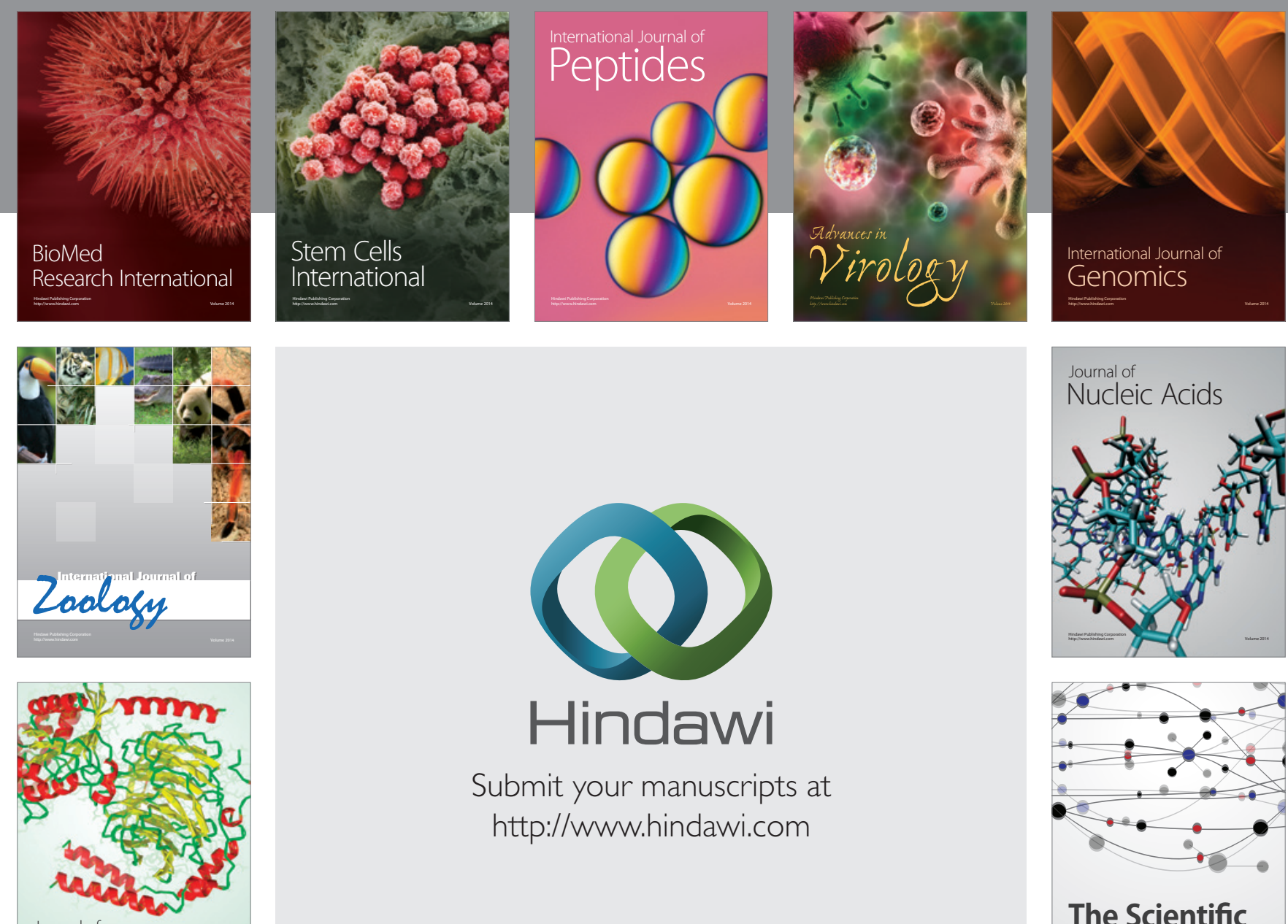

Submit your manuscripts at

http://www.hindawi.com

Journal of
Signal Transduction
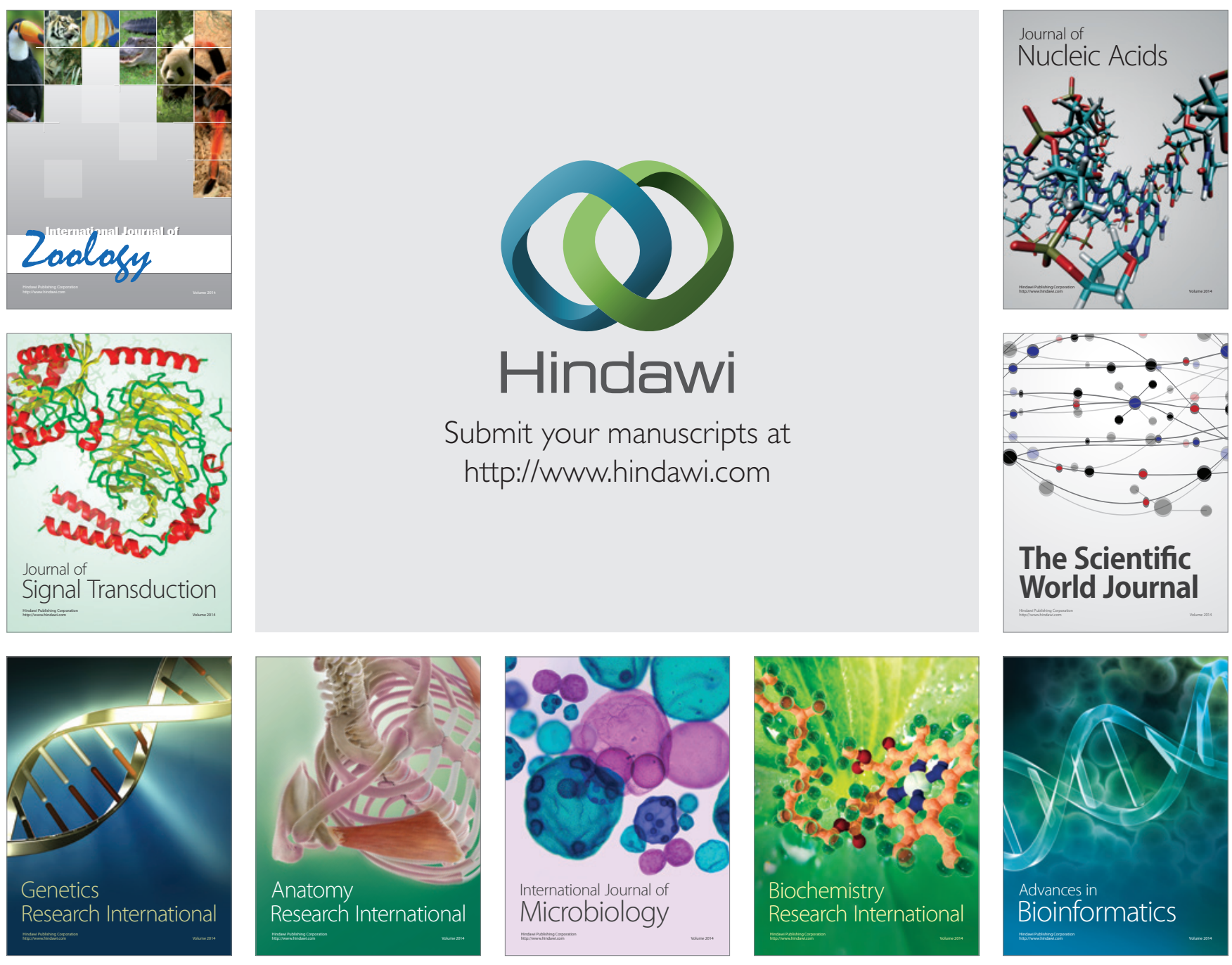

The Scientific World Journal
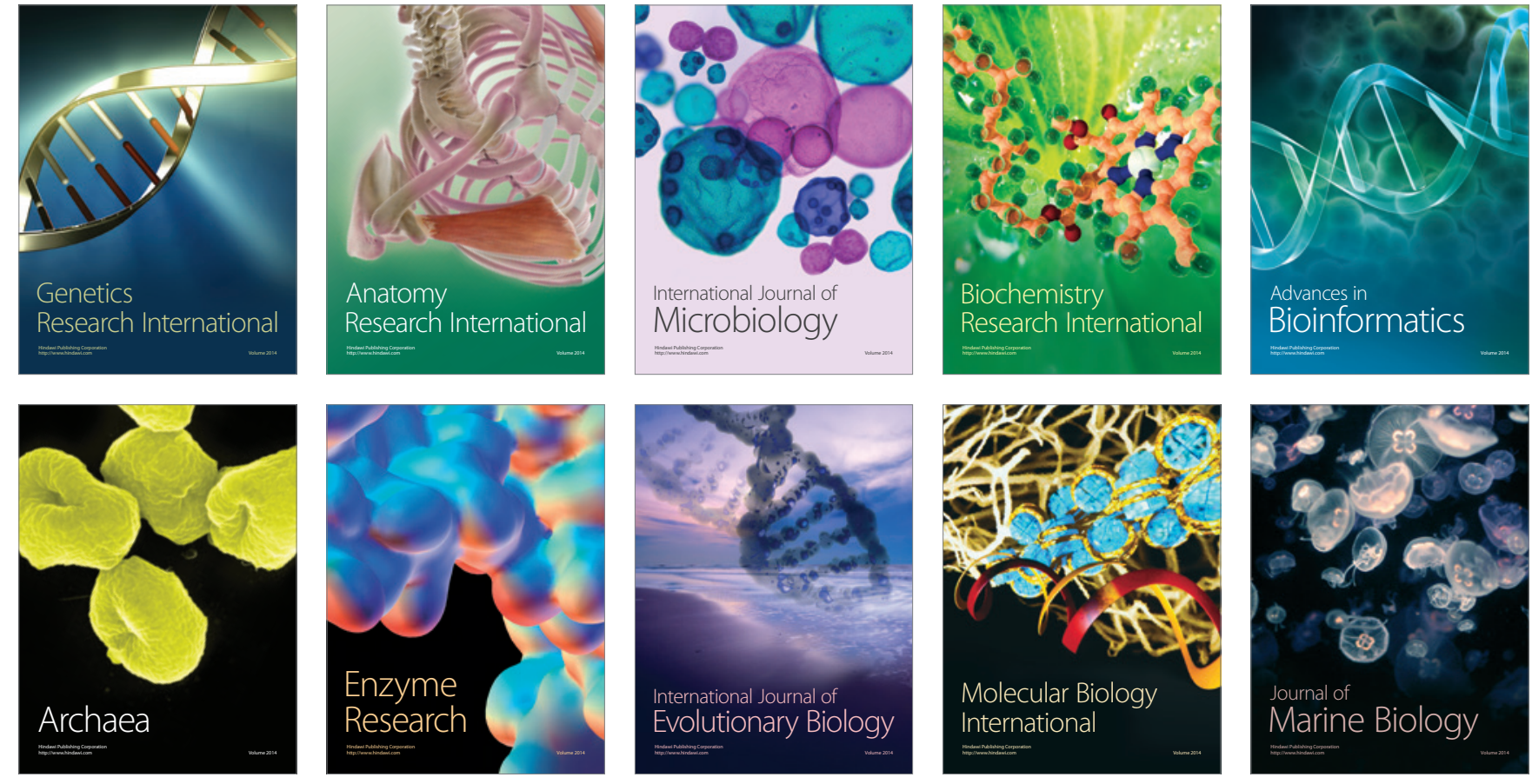